\title{
Topography of Simulated Intestinal Equilibrium Solubility
}

\author{
Claire Dunn, Jeremy Perrier, Ibrahim Khadra, Clive G. Wilson, and Gavin W. Halbert*(1)
}

Strathclyde Institute of Pharmacy and Biomedical Sciences, University of Strathclyde, 161 Cathedral Street, Glasgow G4 0RE, United Kingdom

\section{Supporting Information}

ABSTRACT: Oral administration of a solid dosage form requires drug dissolution in the gastrointestinal tract before absorption. Solubility is a key factor controlling dissolution, and it is recognized that, within the intestinal tract, this is influenced by the luminal fluid $\mathrm{pH}$, amphiphile content, and composition. Various simulated intestinal fluid recipes have been introduced to mimic this behavior and studied using a range of different experimental techniques. In this article, we have measured equilibrium solubility utilizing a novel four component mixture design (4CMD) with biorelevant amphiphiles (bile salt, phospholipid, oleate, and monoglyceride) within a matrix of three $\mathrm{pH}$ values $(5,6$, and 7$)$ and total amphiphile concentrations (11.7, 30.6, and $77.5 \mathrm{mM})$ to provide a topographical and statistical overview. Three poorly soluble drugs representing acidic (indomethacin), basic (carvedilol), and neutral (fenofibrate) categories have been studied. The macroscopic solubility behavior agrees with literature and exhibits an overall increasing solubility from low $\mathrm{pH}$ and total amphiphile concentration to high $\mathrm{pH}$ and total amphiphile concentration. Within the matrix, all three drugs display different topographies, which can be related to the statistical effect levels of the individual amphiphiles or amphiphile interactions on solubility. The study also identifies previously unreported three and four way factor interactions notably between bile salt, phospholipid, $\mathrm{pH}$, and total amphiphile concentration. In addition, the results also reveal that solubility variability is linked to the number of amphiphiles and the respective ratios in the measurement fluid, with the minimum variation present in systems containing all four amphiphiles. The individual 4CMD experiments within the matrix can be linked to provide a possible intestinal solubility window for each drug that could be applied in PBPK modeling systems. Overall the approach provides a novel overview of intestinal solubility topography along with greater detail on the impact of the various factors studied; however, each matrix requires 351 individual solubility measurements. Further studies will be required to refine the experimental protocol in order the maximize information garnered while minimizing the number of measurements required.

KEYWORDS: solubility, four component mixture design, $p H$, total amphiphile concentration, Biopharmaceutics Classification System, FaSSIF, FeSSIF, IVIVC

\section{INTRODUCTION}

Oral administration of solid dosage forms is simple and convenient, and therefore, it is the predominant route of drug administration. Despite the routine process of oral administration, the drug must be absorbed from the gastrointestinal tract into the circulation if systemic therapeutic effects are to occur. The rate and extent of drug absorption is influenced by a multitude of factors related to the drug's physicochemical properties, the formulation, gastro-intestinal tract physiology, and the patient, with respect to food intake or clinical status. ${ }^{1}$ A preeminent factor is the drug's aqueous solubility, since solid drug is not absorbed, and therefore dissolution, which is in part controlled by solubility, ${ }^{2}$ has to occur after the administration of a solid dosage form. The importance of aqueous solubility has been recognized in the Biopharmaceutics Classification System (BCS), ${ }^{3}$ which categorizes drugs based on either a high or low solubility with respect to dose. Low aqueous solubility drugs present a problem during oral administration, since solubility may impact drug absorption and is an increasing issue in drug development due to the proliferation of low solubility compounds. ${ }^{4}$ However, it has been recognized, that a simple aqueous assessment does not always reflect gastrointestinal solubility ${ }^{5}$ due to the presence of multiple components (for example bile salts) or physiological conditions (for example $\mathrm{pH}$ ) which influence solubility.

In order to measure gastrointestinal solubility, several investigators have developed approaches to sample human intestinal fluid (HIF) ${ }^{6}$ and to determine the solubility of drugs in these fluids. ${ }^{7,8}$ These early forays have been expanded, and multiple studies have been conducted to determine the solubility of a range of drugs ${ }^{9,10}$ in both fasted ${ }^{11}$ and fed ${ }^{12}$

Received: November 30, 2018

Revised: February 22, 2019

Accepted: March 8, 2019

Published: March 8, 2019 
HIF samples. These studies have also demonstrated the variability of HIF composition between individuals ${ }^{13}$ and also between anatomical locations within the intestinal tract. ${ }^{14-16}$ In solubility studies, however, this variability can be normalized or reduced, since HIF samples may be pooled or combined to overcome HIF sample volume limitations. ${ }^{9,10,12,14,17}$ Solubility studies in HIF, although the most relevant measurement fluid, are therefore hampered by the anatomical difficulty of sampling, sample volume, and the inherent variability.

In order to mitigate the issues associated with HIF, simulated intestinal fluids (SIF) have been developed, employing physiologically relevant conditions (e.g., $\mathrm{pH})^{10,15}$ and component concentrations (e.g., bile salt and lecithin). ${ }^{18,19}$ These initial SIF media recipe studies have been modified by multiple groups to improve solubility performance with respect to HIF (for tables of fasted and fed recipes see refs 20-22); for example, a recent paper presented five literature versions of fasted SIF and proposed a further modification based around altered bile salt and phospholipid concentrations and surface tension measurements. ${ }^{23}$ In this study, a solubility comparison of ten poorly soluble drugs in three different media, including the proposed version, indicated that, for eight drugs, significant solubility differences between medias were present. Although it was reported that solubility values in the proposed media were closer to literature solubility values in HIF samples.

Statistical design of experiment (DoE) investigations into the solubility contribution of eight typical SIF media components included in either fasted ${ }^{24}$ or fed $^{25}$ recipes indicated that solubility differences of up to several orders of magnitude were possible, even with biorelevant component concentrations. $^{26}$ These studies highlighted that media $\mathrm{pH}$ is a major solubility driver for ionizable acidic drugs, and for poorly soluble basic or nonionizable drugs, four biorelevant amphiphiles (bile salt, phospholipid, oleate, and monoglyceride) were of equal importance to $\mathrm{pH}$. The studies also suggested interesting drug specific solubility variations induced by media components, ${ }^{24,25}$ and that other components (buffer, salt, and pancreatin) did not impact solubility unless specific interactions were present. However, the DoE approach did not visualize the subtle interplay of component concentrations and ratios on solubility and potentially induced variability due to the statistical design.

Solubility determination in a single intestinal media composition, either HIF or SIF, only provides a single point solubility measurement and cannot capture the inherent in vivo variability of intestinal fluid composition and its impact on solubility. The solubility of drugs is known to vary by almost an order of magnitude between different simulated media recipes, ${ }^{23,27}$ but these only represent measurements around a center point of possible compositional variation. Some studies have tackled the influence of media composition on solubility by studying the variation of single factors within the system. The solubility of danazol, for example, has been linearly related to bile salt concentration ${ }^{8}$ in sampled HIF, and variation in cholesterol concentration in a simulated media can either have no impact, increase, decrease, or induce a minima in the solubility of carbamazepine, fenofibrate, danazol, and griseofulvin, respectively. ${ }^{28}$ The application of statistical analysis methods is also possible, ${ }^{24-26}$ with one study stating that there was a "complex interplay" of factors involved. ${ }^{12}$

The DoE studies mentioned above determined that in simulated media, $\mathrm{pH}$ and the four amphiphilic components (sodium oleate (SO), phospholipid (soya phosphatidyl choline
(SPC)), bile salt (sodium taurocholate (NATC)), and monooleate (glyceryl mono-oleate (GMO))) were major factors influencing solubility. In mixture design experiments, the mixture components represent independent factors expressed as fractions of the total sum of the components, which must add to one, with a ternary phase diagram illustrative of a three component system. To visualize the complex solubility interplay between the drug and the amphiphilic components, a four component mixture design (4CMD), which requires a tetrahedron, has been applied to seven poorly soluble BCS class II drugs at a single $\mathrm{pH}$ of 7 and total amphiphile concentration of $11.7 \mathrm{mM} .{ }^{29}$ However, in this study, only a single $\mathrm{pH}$ (7) was employed to ensure that, within the system, SO remained ionized and also only a single total amphiphile concentration $(11.7 \mathrm{mM})$ representitive of the fasted state. The results provided a visualization of solubility behavior within the 4CMD space, indicating that all the drugs displayed different solubility patterns with different minima and maxima, including the number of maxima, and the relative proximities of low and high solubility zones.

It is known, that $\mathrm{pH}$ and amphiphile concentrations vary along the length of the intestinal tract coupled with intra- and interindividual variations. ${ }^{14-16}$ In order to determine if the solubility complexity demonstrated using a single 4CMD condition ${ }^{25}$ is retained when $\mathrm{pH}$ and amphiphile concentration varies, we have extended the 4CMD approach to cover additional $\mathrm{pH}$ (5 and 6) and total amphiphile concentration

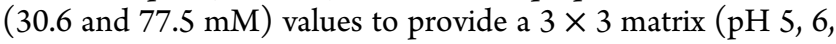
and 7 ; total amphiphile concentration 11.7, 30.6, and 77.5 $\mathrm{mM}$ ) that spans the likely range of biorelevant intestinal values or conditions. This approach fixes the $\mathrm{pH}$ and total amphiphile concentrations, thus permitting an analysis of the impact of the amphiphile ratio on equilibrium solubility under multiple conditions. This has been applied to representative BCS Class II drugs (Table S1) with an acidic (indomethacin), basic (carvedilol), and neutral (fenofibrate) example. Our previous DoE studies ${ }^{24,25}$ indicate that these categories of drugs are likely to exhibit different behaviors in this system based on the $\mathrm{pH}$ and amphiphiles. This will provide a direct visualization of the solubility profile across intestinal media component space; permit comparison of the influence of total amphiphile concentration, amphiphile ratio, and $\mathrm{pH}$ on solubility topography; along with the statistical significance of each amphiphile and their interactions.

\section{MATERIALS AND METHODS}

Materials. Hydrochloric acid $(\mathrm{HCl})$, potassium hydroxide $(\mathrm{KOH})$, acetic acid, sodium dihydrogen orthophosphate $\left(\mathrm{NaH}_{2} \mathrm{PO}_{4}\right)$, sodium chloride $(\mathrm{NaCl})$, chloroform, indomethacin, and fenofibrate were sourced from Sigma-Aldrich, UK. Carvedilol was kindly provided through the OrBiTo initiative by Dr. R. Holm, Head of Preformulation, Lundbeck, Denmark. NATC was purchased from Sigma-Aldrich. Lecithin S PC (phosphatidylcholine from soybean "98\%", SPC) was purchased from Lipoid, Germany. SO was obtained from $\mathrm{BDH}$ Chemical Ltd., Poole England. GMO was a gift from Croda International. All water used was ultrapure Milli-Q water. Methanol and acetonitrile were HPLC grade (VWR, $\mathrm{UK})$, and ammonium acetate was obtained from Merck, Germany.

4CMD. The 4CMD was constructed using Minitab 16.0 simplex lattice with four component input. The lattice incorporated 4 amphiphiles (NATC, SPC, SO, and GMO), 
and the design was augmented with axial points, which included points inside the tetrahedron rather than just the surface. On the basis of the published SIF, DoE experiments, ${ }^{24,25}$ and HIF compositions, ${ }^{15} \mathrm{pH}$ values of 5, 6, and 7 and total amphiphile concentrations of 11.7, 30.6, and 77.5 $\mathrm{mM}$ were chosen to cover a range of possible intestinal values. This provided a $3 \times 3$ matrix of $4 \mathrm{CMD}$ experiments, each at a defined $\mathrm{pH}$ and total amphiphile concentration.

Each 4CMD experiment within the matrix was identical (with the exception of $\mathrm{pH}$ and total amphiphile concentration) and contained 39 media compositions, including 5 inside the tetrahedron (Table 1) and 34 on the four surfaces (Figure 1).

\section{Table 1. 4CMD Composition of Internal Points}

\begin{tabular}{llccc} 
& \multicolumn{4}{c}{ component mol \% } \\
\cline { 2 - 5 } media composition & $\begin{array}{c}\text { bile } \\
\text { salt }\end{array}$ & monoglyceride & phospholipid & $\begin{array}{c}\text { sodium } \\
\text { oleate }\end{array}$ \\
$\begin{array}{l}\text { high bile salt } \\
\text { high }\end{array}$ & 12.5 & 12.5 & 12.5 & 12.5 \\
$\begin{array}{l}\text { monoglyceride } \\
\text { high phospholipid }\end{array}$ & 12.5 & 62.5 & 12.5 & 12.5 \\
$\begin{array}{l}\text { high sodium oleate } \\
\text { center point }\end{array}$ & 12.5 & 12.5 & 62.5 & 12.5 \\
& 25 & 25 & 12.5 & 62.5 \\
\end{tabular}

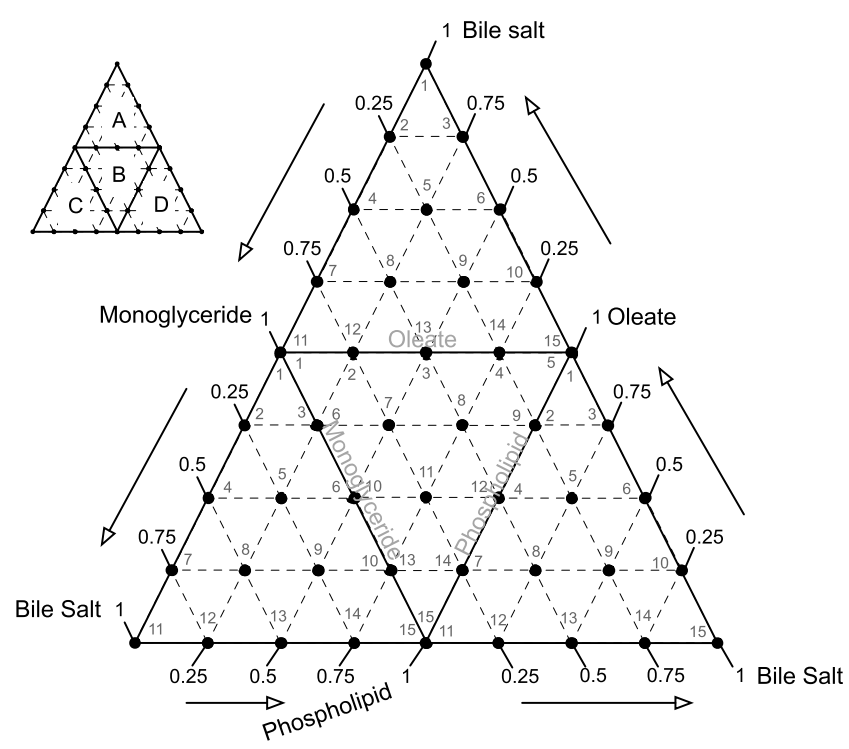

Figure 1. $4 \mathrm{CMD}$ representation of surface points. Flattened representation of the four surfaces of the tetrahedron when open from the top vertex, which is $100 \mathrm{~mol} \%$ bile salt, and the remaining $100 \mathrm{~mol} \%$ vertices are labeled with the appropriate amphiphile.

Each tetrahedron face represented a ternary phase diagram with the absence of one amphiphile, and the four faces are represented as triangle $\mathrm{A}$, no phospholipid NATC/SO/GMO; triangle $\mathrm{B}$, no bile salt $\mathrm{SPC} / \mathrm{SO} / \mathrm{GMO}$; triangle $\mathrm{C}$, no sodium oleate NATC/SPC/GMO; and triangle D, no monoglyceride NATC/SPC/SO. Each face had 15 combinations, with the three points on each edge shared by two faces, and the vertex points shared by three faces. The concentrations are given in mol \% of the total amphiphile molar concentration, either 11.7, 30.6 , or $77.5 \mathrm{mM}$.

Equilibrium Solubility Measurements. Phosphate buffer containing $68 \mathrm{mM} \mathrm{NaCl}$ and $45 \mathrm{mM} \mathrm{NaH} \mathrm{PO}_{4}$ was prepared with deionized water, and the $\mathrm{pH}$ was adjusted to the required $\mathrm{pH}$. Stock solutions of NATC and SO were freshly prepared from solids by being dissolved in the phosphate buffer. The SPC stock solution was prepared by dissolving the lipid in chloroform, removing the chloroform by evaporation under nitrogen, and dispersing the dried SPC film into phosphate buffer. GMO cannot dissolve in buffer, so the stock GMO was prepared by mixing NATC and GMO (1:10.7 ratio) at the required total concentration, and for practical experimental reasons, this solution was employed as $100 \mathrm{~mol} \%$ GMO. The required $4 \mathrm{CMD}$ media was prepared from the stock solutions, and equilibrium solubility was determined as follows.

An excess amount of solid drug $(10 \mathrm{mg})$ was added to $4 \mathrm{~mL}$ of each mixed lipid media in Corning $15 \mathrm{~mL}$ centrifuge tubes and then placed on a rotating wheel mixer for $1 \mathrm{~h}$. After which, if required, the $\mathrm{pH}$ was readjusted to the desired value in order to maintain a constant $\mathrm{pH}$ during the experimental period. Tubes were then placed on the mixer and equilibrated at $37^{\circ} \mathrm{C}$ for $24 \mathrm{~h}$, with $\mathrm{pH}$ being checked after incubation. This time frame and procedure has previously been shown to provide a reproducible determination of equilibrium solubility. ${ }^{24,25,29}$ The saturated supernatant was separated by centrifugation at $13000 \mathrm{rpm}$ for $5 \mathrm{~min}$ and transferred for HPLC analysis using an Agilent Technologies 1260 Series Liquid Chromatography system with Clarity Chromatography software (see Table S2 for analysis details). Each 4CMD experiment was conducted once.

Statistical Analysis and Graphical Presentation. The measured equilibrium solubility data for each 4CMD experiment were statistically analyzed in Minitab 16.0 to calculate the statistical effect values for each amphiphile and amphiphile interaction. The standardized effect value for each amphiphile can be calculated by dividing the coefficient (which represents the change in mean response associated with an increase in that term, while the other terms are held constant) with standard error, which is similar to a $p$-value, and thus indicates the effects in the model that are statistically significant. There is no $p$-value generated for each $100 \mathrm{~mol} \%$ amphiphile (single parameter terms), but only standardized effect values show the magnitude of effect. The standardized effect values of each single amphiphile are closely related with the solubility in the media that has $100 \mathrm{~mol} \%$ of that amphiphile; thus; the higher the value, the higher the solubility within the pure amphiphile. Note, that $\mathrm{pH}$ or total amphiphile concentration are not variables within an individual 4CMD experiment and therefore cannot be reported, other than by comparison across the matrix. The Kolmogorov normality test was used in Minitab to assess the distribution of each 4CMD data set; on the basis of the result that some data sets have a non-normal distribution, the Mann-Whitney test was universally applied to evaluate differences between $4 \mathrm{CMD}$ data sets and the comparator fasted and fed DoE data sets.

Surface ternary contour plots with smoothing (Figure 3) were generated in Matlab R2015a on Mac OSX 10.10.5 using a contour plot from Alchemyst-ternplot-9c72b90. The solubility topography plot (Figure 7) was generated in Matlab R2015a on Mac OSX 10.10.5 using the surface mesh plot function and the center point values from each 4CMD experiment within the matrix. The remaining figures were generated using either Prism 7.0d or DataGraph 4.3 on Mac OSX 10.10.5.

\section{RESULTS}

Measured Equilibrium Solubility Distributions. In Figure 2, the measured equilibrium solubility distributions for each $4 \mathrm{CMD}$ experiment (surface and internal points) 

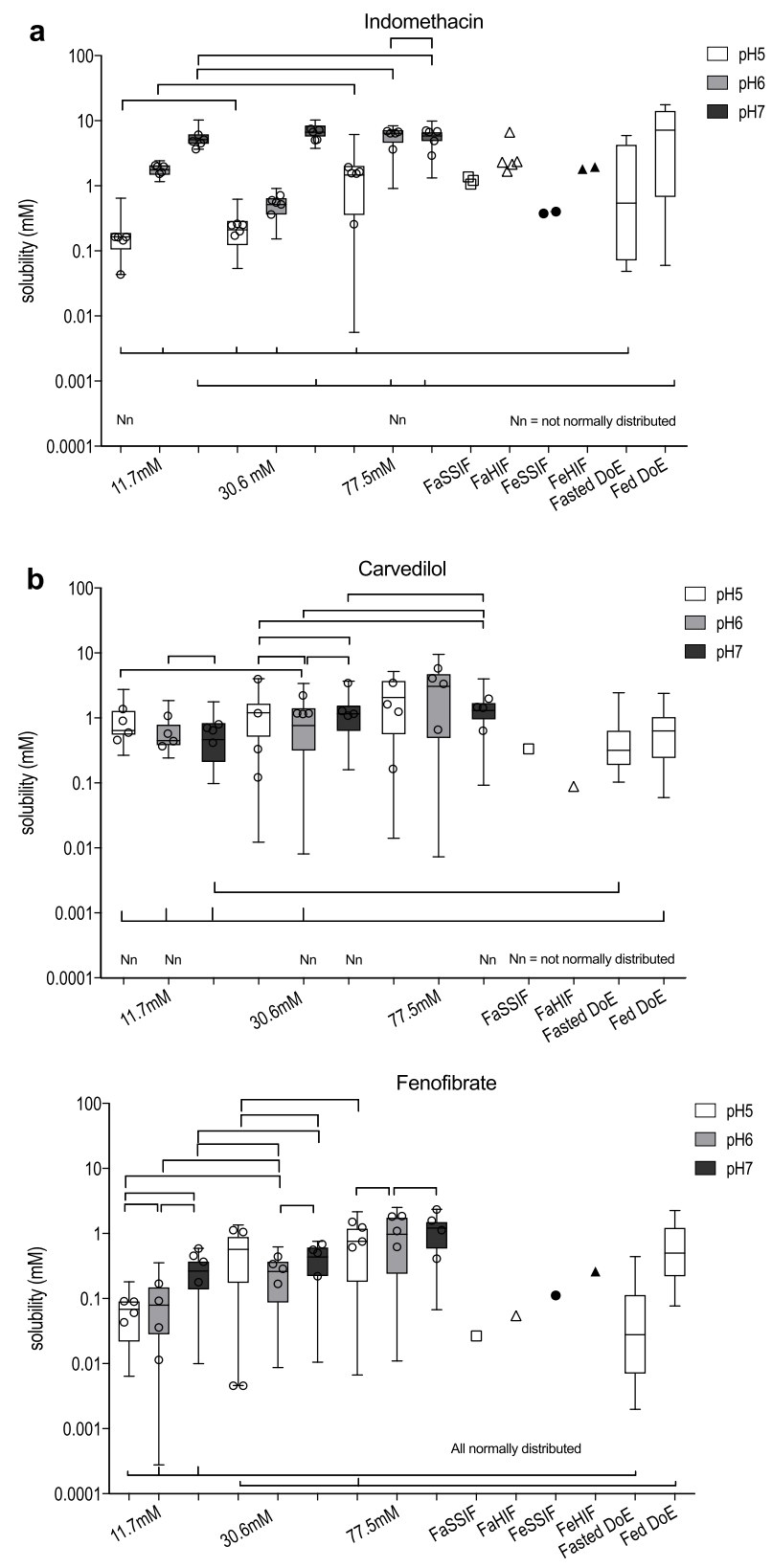

Figure 2. A statistical comparison of measured equilibrium solubility distributions. Measured equilibrium solubility distributions presented as box and whisker plots based on measurement $\mathrm{pH}$ and total amphiphile concentration. Each box and whisker represents all the data measurements from one 4CMD experiment from high to low, maximum value, 75th percentile; median, 25th percentile; and minimum value. Total amphiphile concentration as stated on the $x$ axis. $\mathrm{pH} 5, \square$; pH 6, gray $\square$; and $\mathrm{pH} 7, \mathbf{\square}$. Nn, Data distribution of 4CMD is not normal as assessed by the Kolmogorov normality test Minitab version 16.0. Bars link data sets considered statistically equivalent $(p>0.05)$, Mann-Whitney test, Minitab version 16. $O$, 4CMD internal points which contain all four amphiphiles, see Table 1. Comparative data: FaSSIF, fasted simulated intestinal fluid; FaHIF, fasted human intestinal fluid; FeSSIF, fed simulated intestinal fluid; FeHIF, fed human intestinal fluid. All data were taken with permission from from refs 30 (Copyright 2010 American Chemical Society) and 17 (Copyright 2014 Elsevier). Fasted DoE data were taken with permission from ref 24 (Copyright 2015 Elsevier). Fed DoE data were taken with permission from ref 25 (Copyright 2017 Elsevier). (a) Indomethacin, (b) carvedilol, and (c) fenofibrate. within the matrix are presented as box and whisker plots grouped by $\mathrm{pH}$ and total amphiphile concentration along with reported literature solubility values in both sampled and simulated intestinal fluids ${ }^{17,30}$ and distributions from previous design of experiment studies. ${ }^{24,25}$ For all drugs, the single point literature solubility values from either fasted or fed, simulated or sampled, intestinal fluids, determined by various protocols which are not equivalent to the current study, lie within the envelope of the lowest and highest 4CMD matrix results. The majority of $4 \mathrm{CMD}$ distributions lie within the solubility zone provided by the lowest fasted and highest fed DoE whisker values. There are some exceptions to this with carvedilol, the most obvious producing box distributions that extend above and below the solubility zone, especially at the higher total amphiphile concentrations. For indomethacin (two of nine) and fenofibrate (one of nine), whiskers extend outside the solubility zone to provide lower values than those reported.

Statistical examination of the solubility distributions indicates that some systems are not normally distributed ( $\mathrm{Nn}$ in Figure 2). Especially for carvedilol, where five out of the nine experiments provided non-normal distributions, with only two for indomethacin and zero for fenofibrate. In general, the internal points, which contain all four amphiphiles, are grouped around the $75-25 \%$ distribution box, rather than at the whiskers, although there are exceptions. For indomethacin, one internal point lies on a lower whisker at low $\mathrm{pH}$ and total amphiphile concentration, which is similar for fenofibrate, where two sit on the low whisker at low $\mathrm{pH}$ and total amphiphile concentration of $30.6 \mathrm{mM}$. For carvedilol, two points form whiskers at the total amphiphile concentration of $30.6 \mathrm{mM}$ and $\mathrm{pH} 5$ and 7 , respectively.

Indomethacin demonstrates increasing solubility with increasing $\mathrm{pH}$ within each total amphiphile concentration, with an increased variability, especially to lower values, also related to the total amphiphile concentration. Statistically equivalent indomethacin experiments tend to be grouped based on $\mathrm{pH}$, the low $\mathrm{pH}$, low total amphiphile experiments are equivalent to the fasted $\mathrm{DoE}$ with the high $\mathrm{pH}$, high total amphiphile concentration experiments equivalent to the fed DoE. For carvedilol, there is an apparent small increase in solubility with total amphiphile concentration, and within the lowest total amphiphile concentration $(11.7 \mathrm{mM})$, there is a decrease in solubility with increasing $\mathrm{pH}$. However, it is interesting that the systems at a total amphiphile concentration of $30.6 \mathrm{mM}$ are statistically equivalent to each other and to the experiments at lower or higher total amphiphile concentration. Also the experiments at a total amphiphile concentration of $11.7 \mathrm{mM}$ are equivalent to the fed $\mathrm{DoE}$, with the $\mathrm{pH} 7$ system also equivalent to the fasted DoE, with the only other equivalence being the $\mathrm{pH} 6$ total amphiphile concentration of $30.6 \mathrm{mM}$ to the fed DoE. There is also a marked increase in solubility variability at $\mathrm{pH} 5$ and 6 at total amphiphile concentrations of 30.6 and $77.5 \mathrm{mM}$ that is reduced at the highest $\mathrm{pH}$ tested. Finally, fenofibrate shows a trend of increasing solubility with increasing total amphiphile concentration but no $\mathrm{pH}$ effect, since experiments within a total amphiphile concentration grouping are generally equivalent. This is also reflected in the comparison between individual 4CMD experiments and the previous fasted and fed DoE distributions where, for the latter, generally fasted is equivalent to the low concentration total amphiphile experiments and $\mathrm{pH}$, with the fed being equivalent to the higher concentration total amphiphile experiments and $\mathrm{pH}$. 


\section{Calculated Surface Equilibrium Solubility Contour}

Plots. Figure 3 presents the calculated surface equilibrium solubility contour plots as a $\mathrm{pH}$ and total amphiphile concentration matrix. The color shades indicate solubility
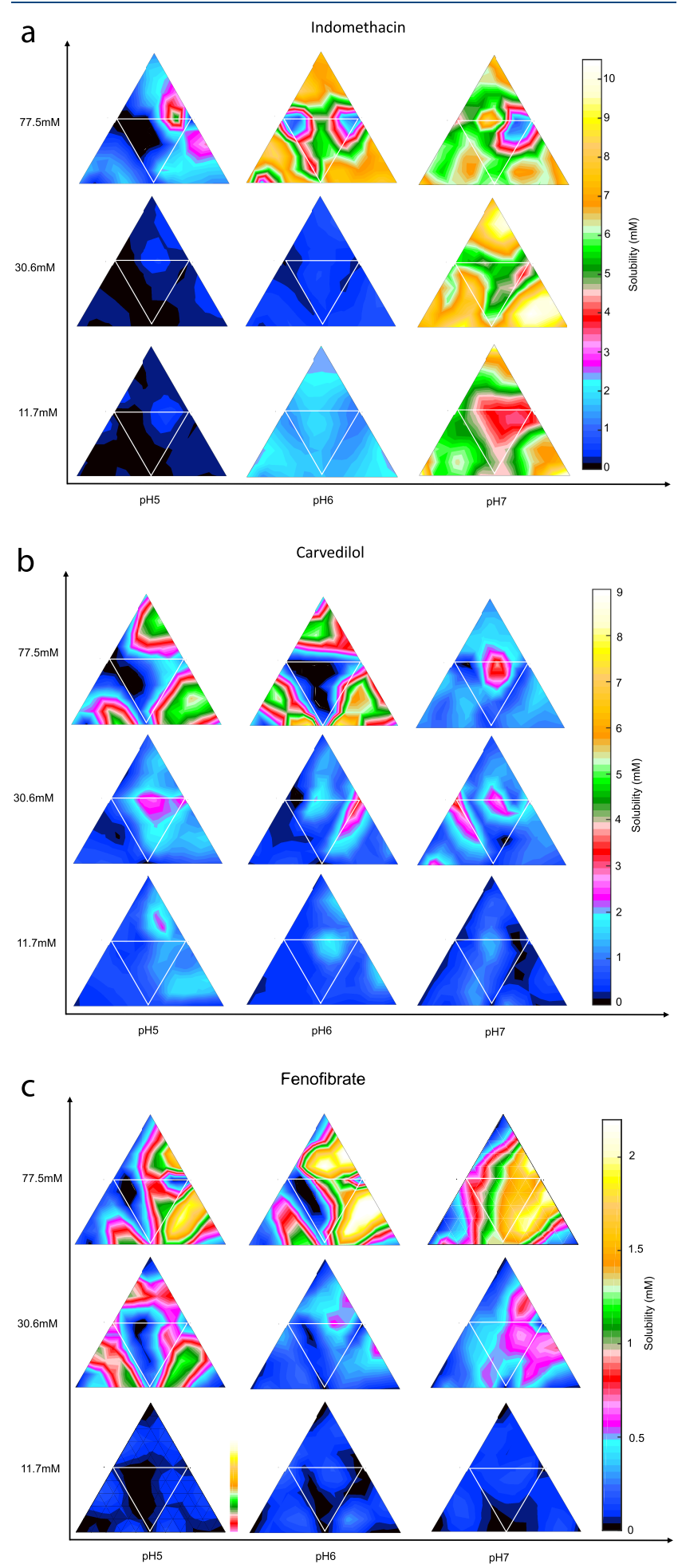

Figure 3. Calculated equilibrium solubility contour plots presented as a matrix based on measurement $\mathrm{pH}$ and total amphiphile concentration. The color shades attached to individual figures represent the calculated solubility contour concentration (mM) values for each drug. $\mathrm{NB}$, the solubility ranges vary between figures. (a) Indomethacin, (b) carvedilol, and (c) fenofibrate. values, with yellow representing the highest solubility and black/dark blue the lowest. Note, solubility ranges vary between figures, while the color scheme is constant.

It is evident that each drug has a unique profile, either at any individual 4CMD experiment or across the whole matrix, no drugs have identical high solubility zones, and also, the extent and variability of solubility is different for each drug. Triangle B does seem to have a greater involvement in low solubility zones when compared to the remainder of the tetrahedron. Overall, variation in solubility is greater than the previous study, ${ }^{29}$ but this is to be expected with an increased $\mathrm{pH}$ and total amphiphile concentration range. Generally, solubility for all three drugs is lowest at the lower left 4CMD experiment at $\mathrm{pH}$ 5 and total amphiphile concentration of $11.7 \mathrm{mM}$ and increases to the top right. However, the magnitude of this increase varies between the drugs. Carvedilol (Figure $3 \mathrm{~b}$ ) behaves differently since the highest solubility is at $\mathrm{pH} 6$ and $77.5 \mathrm{mM}$.

For indomethacin (Figure 3a) at $\mathrm{pH} 5$ and a total amphiphile concentration of $11.7 \mathrm{mM}$, there is a high solubility zone situated at the junction of triangles $\mathrm{A}$ and $\mathrm{B}$ and a low solubility zone around the junction of triangles B and C. These zones remain in approximately the same position as the total amphiphile concentration increases with a slight movement of the high solubility zone to the junction of triangles $A, B$, and D. A similar change is seen with the systems at $\mathrm{pH} 6$ and 7 as the total amphiphile concentration is increased, although the zones of high and low solubility are in different locations. At 11.7 $\mathrm{mM}$, the topography changes as $\mathrm{pH}$ increases, and the low solubility zone in triangles $\mathrm{B}$ and $\mathrm{C}$ disappears or shifts to the junction of triangles $\mathrm{A}, \mathrm{B}$ and $\mathrm{D}$, with a $\mathrm{pH}$ induced increase in overall solubility. For carvedilol (Figure $3 \mathrm{~b}$ ) at $\mathrm{pH} 5$ and a total amphiphile concentration of $11.7 \mathrm{mM}$, the high solubility zone situated within triangle $\mathrm{A}$ and also across triangle $\mathrm{D}$ shifts position slightly with a constant $\mathrm{pH}$, but increasing the total amphiphile concentration to $77.5 \mathrm{mM}$ causes three high solubility zones in triangles $\mathrm{A}, \mathrm{C}$, and $\mathrm{D}$. At $\mathrm{pH} 6$ with an increasing total amphiphile concentration, there are similarities in behavior with the initial high solubility point situated between triangle $\mathrm{B}$ and $\mathrm{D}$ with a shift as the total amphiphile concentration increases to high solubility zones at the vertex of triangles $\mathrm{A}, \mathrm{C}$, and $\mathrm{D}$ and the low solubility zone in triangle $\mathrm{B}$. At $\mathrm{pH} \mathrm{7,} \mathrm{the} \mathrm{profile} \mathrm{is} \mathrm{different,} \mathrm{with} \mathrm{the} \mathrm{high} \mathrm{solubility} \mathrm{zone} \mathrm{at}$ a total amphiphile concentration of $77.5 \mathrm{mM}$ in a similar location to the zone determined at $\mathrm{pH} 5$ and total amphiphile concentration of $30.6 \mathrm{mM}$ and no low solubility zone in triangle $\mathrm{B}$. With constant total amphiphile concentration but variable $\mathrm{pH}$, the profiles are different; for example, triangle A exhibits consistent behavior across the $\mathrm{pH}$ ranges at 11.7 and $30.6 \mathrm{mM}$ but a very different behavior at a total amphiphile concentration of $77.5 \mathrm{mM}$. At pH 5 and 6 and total amphiphile concentration of $77.5 \mathrm{mM}$, the highest solubility is at the vertices of the main triangle (A, B, C, and D combined) but shifts away from these vertices at $\mathrm{pH}$ 7. For fenofibrate (Figure 3c) at $\mathrm{pH} 5$ and total amphiphile concentration $11.7 \mathrm{mM}$, there are high solubility zones situated in triangles A, C, and D. If $\mathrm{pH}$ is constant, these zones remain in the same position with increasing total amphiphile concentration, although a degree of variation is induced as the solubility increases, especially at the vertex of triangle $A, B$, and C. Similar behavior is evident at $\mathrm{pH}$ 6 and 7 as the total amphiphile concentration is increased, although there are changes in the starting locations of the zones. If $\mathrm{pH}$ is changed at a constant total amphiphile 


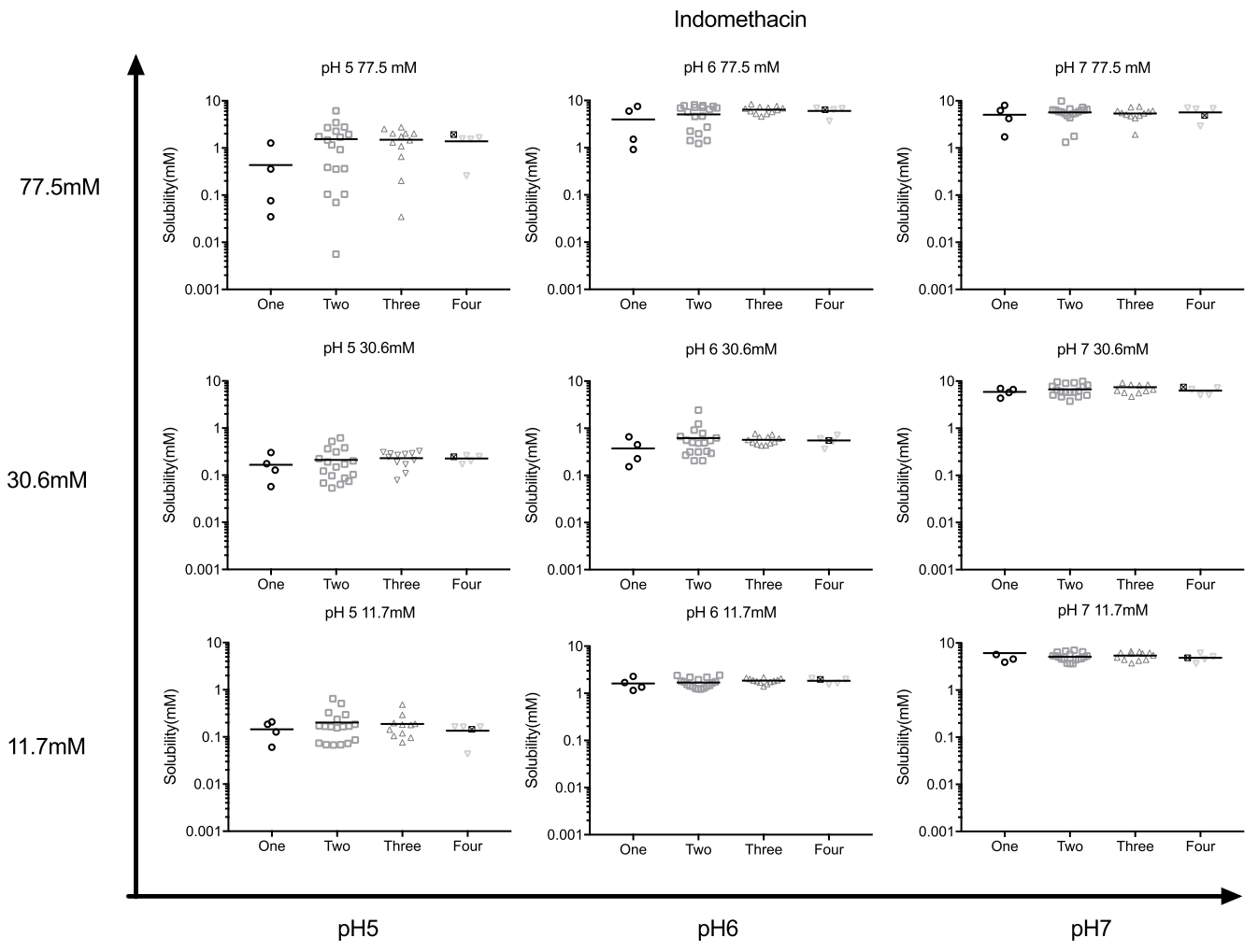

Figure 4. Influence of amphiphile number on measured equilibrium solubility. Indomethacin equilibrium solubility data points determined during the 4CMD experiment presented as individual points based on the number of amphiphiles present. (Bar) The mean for each individual data set. (•) In the four amphiphile columns = center point (see Table 1). See the Supporting Information for carvedilol and fenofibrate figures.
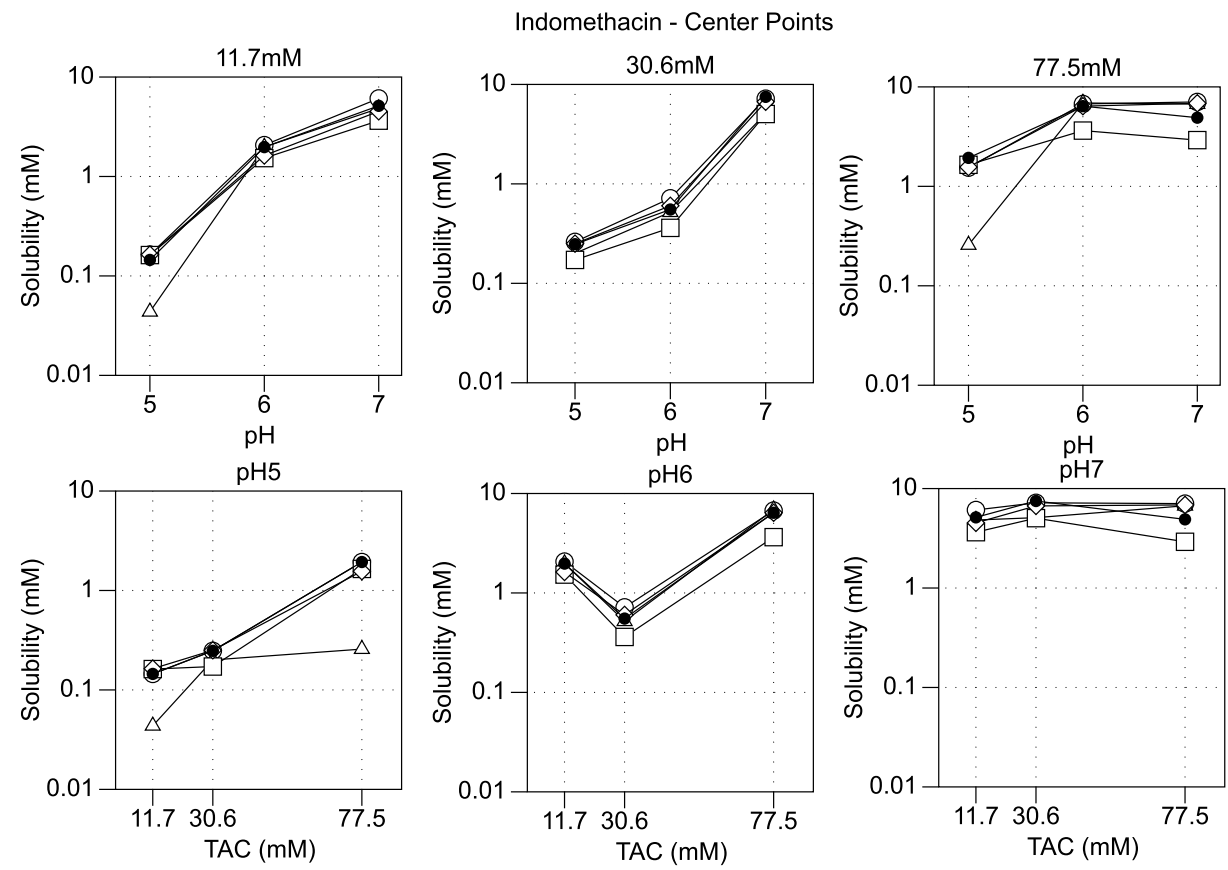

Figure 5. Internal point results. Indomethacin equilibrium solubility data point plots determined during the 4CMD experiment. Data are presented as a matrix based on measurement $\mathrm{pH}$ and total amphiphile concentration. Each individual plot represents the internal data points (see Table 1) from the 4CMD experiments grouped either by measurement $\mathrm{pH}$ or total amphiphile concentration. NB, The surface data points are not included in this analysis. ๑, Center point; $\bigcirc$, high NATC; $\triangle$, high SO; $\diamond$, high GMO; and $\square$, high SPC. See the Supporting Information for the carvedilol and fenofibrate figures.

concentration, the results are different with some interesting variations between the amphiphile concentrations. At 11.7 $\mathrm{mM}$, the low solubility zone in triangle $\mathrm{B}$ moves to the junction of triangles B, C, and D, which is low NATC and SO but high GMO. At a total amphiphile concentration of $30.6 \mathrm{mM}$, there is an overall lower solubility associated with $\mathrm{pH} 6$ and a similar 
(to $11.7 \mathrm{mM}$ ) shift of the low solubility zone, although this looks simply to be due to the overall increase in solubility at the highest $\mathrm{pH}$. The highest total amphiphile concentration $(77.5 \mathrm{mM})$ is similar to the high solubility zones remaining in the same location and a loss of the low solubility zone, which looks to be simply due to an increased overall solubility as $\mathrm{pH}$ increases.

Impact of Amphiphile Number on Measured Equilibrium Solubility. Each 4CMD experiment consists of 39 measurement points containing either one, two, three, or four amphiphiles, and data for indomethacin are presented in Figure 4 (see Figure S1a,b). The results indicate that, in the majority of cases, the solubility variability is largest with the one or two amphiphile systems, reduced in the three amphiphile system, and smallest when all four amphiphiles are present. In addition, the center point in the majority of cases is very close to the mean value for the four amphiphile systems. As before, there are some exceptions to these general comments; for example, indomethacin at $\mathrm{pH} 5$ and total amphiphile concentration of $11.7 \mathrm{mM}$, where one of the internal points (high oleate) has the lowest solubility, and carvedilol at $\mathrm{pH} 5$ and total amphiphile concentration of 30.6 $\mathrm{mM}$, where the four amphiphile system exhibits a large variability.

Internal Point-Measured Equilibrium Solubility Plots. Each 4CMD tetrahedron contains five internal points, which, based on the previous results (Figure 4), exhibit greater consistency and are presented separately for indomethacin in Figure 5 (see Figure S2a,b). For indomethacin, the $\mathrm{pH}$ dependency of solubility is again evident with the reduced influence of total amphiphile concentration at higher $\mathrm{pH}$. However, the extreme solubility points presented in the whiskers are missing, especially at $\mathrm{pH} 5$ and the $77.5 \mathrm{mM}$ total amphiphile concentration. The carvedilol profile exhibits a greater variability of behavior but is similar to generally increasing solubility with total amphiphile concentration, but particular systems show unusual behavior; for example, the high oleate ratio exhibits a marked $\mathrm{pH}$ dependency at a total amphiphile concentration of 30.6 and $77.5 \mathrm{mM}$. Fenofibrate is again similar to the total analysis, with generally increasing solubility with increasing total amphiphile concentration but with some unusual variation. There is an extremely low solubility measured at $\mathrm{pH} 5$ and a total amphiphile concentration of $30.6 \mathrm{mM}$ for the high NATC and GMO systems.

Statistical Factor Analysis. For indomethacin, the highest single effect value (Figure 6, see Figure S3a,b,c,d) is for NATC, followed in roughly equivalent importance by GMO, SPC, and $\mathrm{SO}$, especially at a low $\mathrm{pH}$ and the higher total amphiphile concentration. As the total amphiphile concentration increases, the magnitude of the effect values decrease and even becomes negative for $\mathrm{SO}$ at $\mathrm{pH} 5$ and a total amphiphile concentration of $77.5 \mathrm{mM}$. The carvedilol single effect values are more varied but also show a decreasing significance as the total amphiphile concentration increases but with no apparent $\mathrm{pH}$ influence. For fenofibrate, NATC shows a negative impact on solubility in seven out of the nine systems, with SO generally being the largest positive factor, except at $\mathrm{pH} 7$ and the $77.5 \mathrm{mM}$ total amphiphile concentration, where the remaining amphiphiles exert a strong positive effect.

For indomethacin, 14 significant factor interactions (Figure $6 \mathrm{~b}$ ) are present out of a possible total of 54, with five out of a possible 18 at a total amphiphile concentration of $11.7 \mathrm{mM}$,

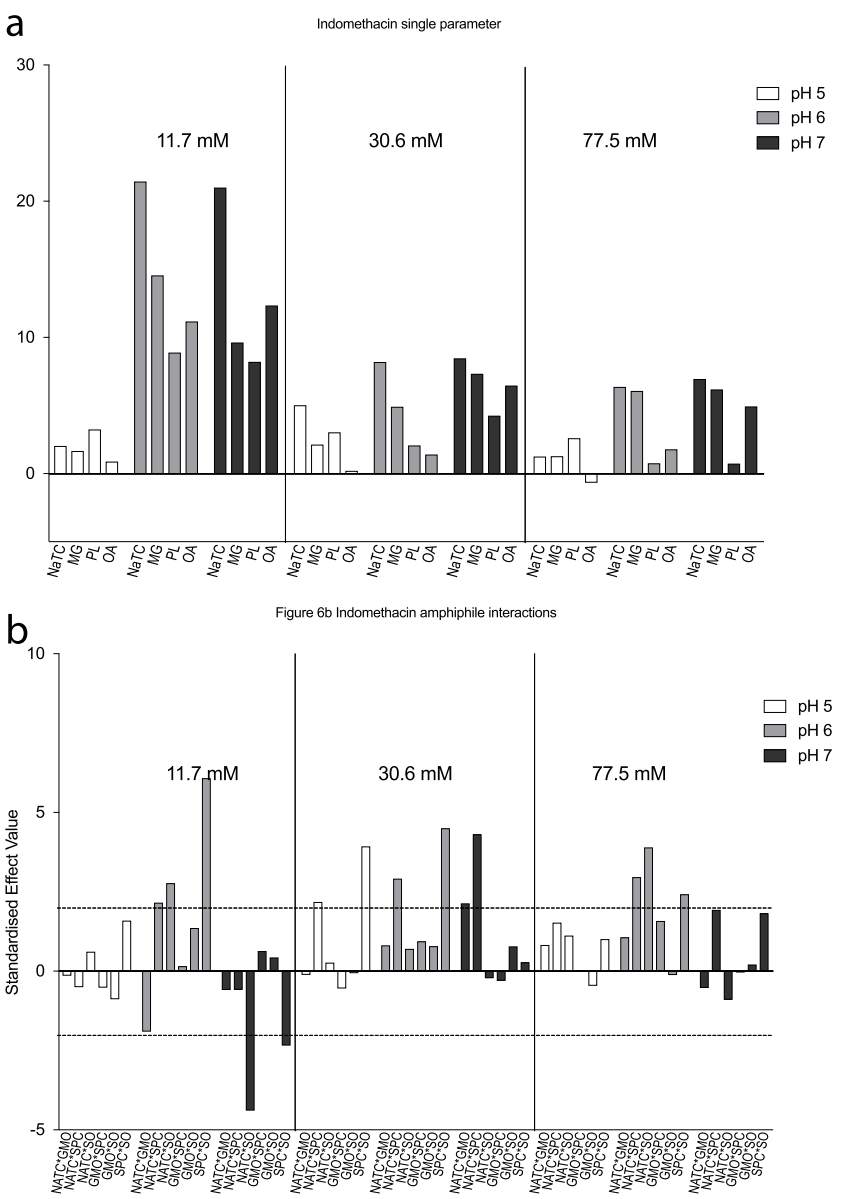

Figure 6. Indomethacin standardized effect values for individual amphiphiles and two amphiphile interactions. Indomethacin standardized effect value for individual amphiphiles and amphiphile combinations calculated by dividing coefficient with standard error; the dashed line indicates the $p$-value $(p>0.05)$. Due to model limitations, no $p$-value was generated for single amphiphile terms, only a standardized effect value. The bars $>0$ indicate a positive solubility effect, and the bars $<0$ indicate a negative solubility effect. NaTC, bile salt; GMO, monoglyceride; SPC, phospholipid; and SO, sodium oleate. (a) Indomethacin single standardized effect values; (b) indomethacin two amphiphile interaction standardized effect values. See the Supporting Information for the carvedilol and fenofibrate figures.

decreasing to three at the $77.5 \mathrm{mM}$ total amphiphile concentration. The majority of the interactions are positive, but two negative interactions are present between NATC and SO and SPC and SO at pH 7 and the lowest total amphiphile concentration of $11.7 \mathrm{mM}$. For carvedilol, the number of significant interactions is similar at 12 , but the incidence is different with six out of eight present at the $77.5 \mathrm{mM}$ total amphiphile concentration and no negative solubility interactions. No consistent pattern is evident in the significant interactions, other than they seem to be greater at the lower $\mathrm{pH}$ values of the highest total amphiphile concentration. At the highest total amphiphile concentration of $77.5 \mathrm{mM}$, the interaction between bile salt and monoglyceride and bile salt and phospholipid are the largest significant interactions at $\mathrm{pH}$ 5 , reduced at $\mathrm{pH} 6$, and not significant at $\mathrm{pH} 7$; this indicates the detection of a three way interaction between $\mathrm{pH}$ with NATC and either SPC and GMO. Fenofibrate has the highest number of significant interactions at 23 , with a consistent 
response since NATC and SPC positively interact in eight out of the nine experiments. In addition, there is a pattern that the interactions have a greater magnitude as the total amphiphile concentration increases, but at the highest concentration, this is reduced by the highest $\mathrm{pH}$. This indicates that, for NATC with GMO or SPC and GMO with SPC, there is a detection of a possible three way interaction with a $\mathrm{pH}$ at the highest total amphiphile concentration.

Topography of Simulated Intestinal Equilibrium Solubility. The center points in each 4CMD experiment represent an equilibrium solubility determination at a particular $\mathrm{pH}$ and total amphiphile concentration and therefore can be plotted along with a calculated solubility gradient between the points to provide a visualization of the solubility topography (Figure 7). Indomethacin exhibits an increased solubility with increased $\mathrm{pH}$ but with an interesting trough at $\mathrm{pH} 6$ and a total amphiphile concentration of $30.6 \mathrm{mM}$, and it also has a reduced solubility at high $\mathrm{pH}$ due to high total amphiphile concentration reducing solubility. Carvedilol has a different topography with increasing solubility, due to increasing the total amphiphile concentration, and very little $\mathrm{pH}$ effect, except at $\mathrm{pH} 7$ and the highest total amphiphile concentration. The shape of the topography indicates that this is a different effect to that seen with indomethacin. Fenofibrate also displays a different topography with generally increasing solubility with increasing total amphiphile concentration but with a $\mathrm{pH}$ induced valley at $\mathrm{pH} 6$ and total amphiphile concentration of $30.6 \mathrm{mM}$ and a peak at $\mathrm{pH} 6$ and $77.5 \mathrm{mM}$ total amphiphile concentration.

Intestinal Equilibrium Solubility Window. The previous results are presented in rank order based on the two main study variables; however, within the gastrointestinal tract, this order is irrelevant, and it is known that $\mathrm{pH}$ and amphiphile concentration vary as the material passes down the tract; ${ }^{12,15,16}$ for example, bile salt is reabsorbed as it moves through the small intestine. To gauge the possible impact of these media changes on solubility, Figure 8 presents the span between the minimum and maximum solubility values of the high ratio internal points and the center point at a selection of 4CMD conditions. The starting point is low $\mathrm{pH}$ and total amphiphile concentration (see Figure 8a) reflective of proximal small intestine post gastric transit, followed by rising $\mathrm{pH}$ and total amphiphile concentration, as the material transits and bile is excreted, and then a reduction in total amphiphile concentration without a major change in $\mathrm{pH}$ to mimic the distal small intestine post absorption. The authors recognize that this construct is artificial, but it is similar to research on early, middle, and late SIFs ${ }^{20}$ and therefore has a precedent.

The drugs produce three different profiles, and indomethacin (Figure $8 \mathrm{~b}$ ) has an initial low solubility which increases as the $\mathrm{pH}$ increases and is not reduced by the lower total amphiphile concentrations in the latter analysis stages. The initial stages provide a solubility window that is lower than the required high solubility value for BCS Class I, but once the $\mathrm{pH}$ is above 6 , all conditions meet the high solubility limit. For carvedilol (Figure 8c), a more constant solubility profile, with an approximate center around $1 \mathrm{mM}$ throughout the entire space, is evident. The result indicates that, based on a $25 \mathrm{mg}$ dose, carvedilol solubility is counterintuitively in the high solubility range throughout the analysis space. Fenofibrate (Figure $8 \mathrm{~d}$ ) has a different profile with an initial low solubility which rises to a maxima at the highest $\mathrm{pH}$ and total amphiphile concentration and then decreases as total amphiphile
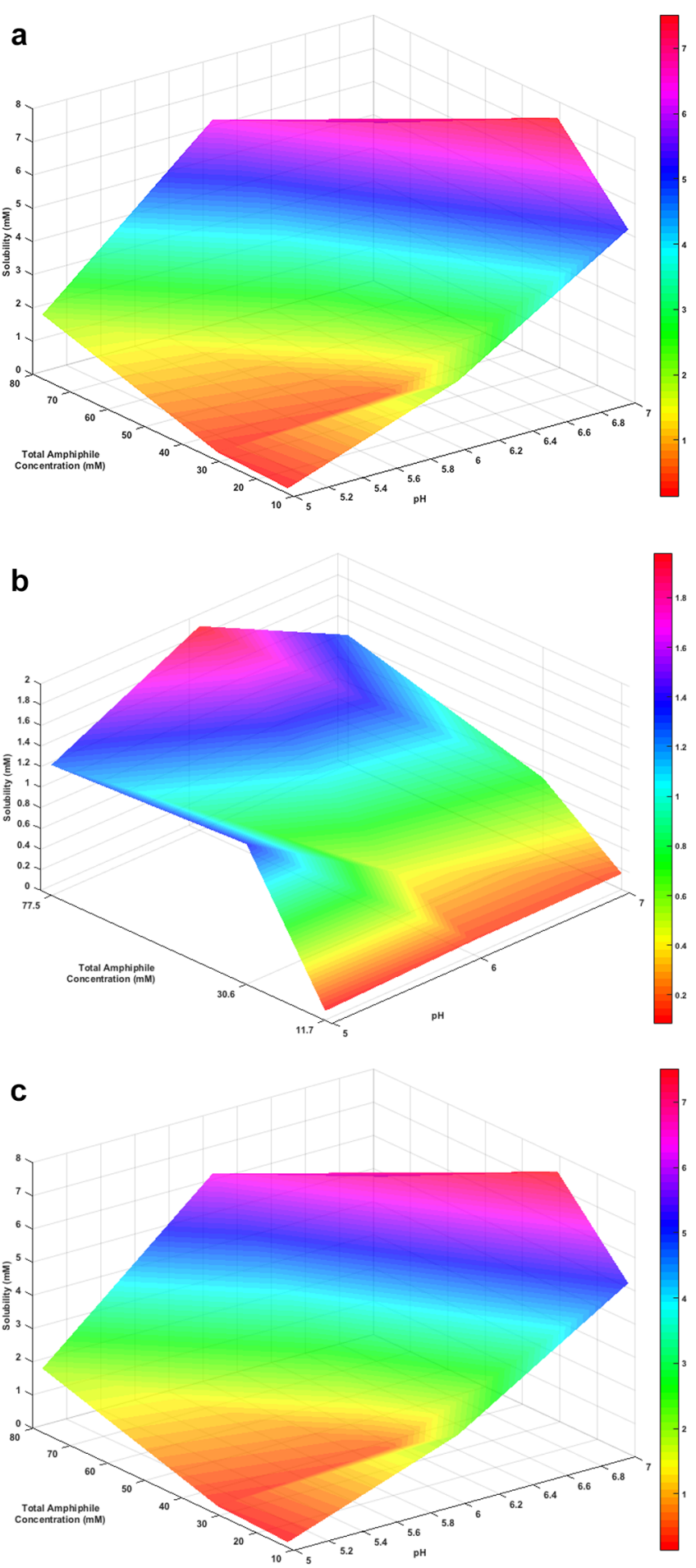

Figure 7. The topography of intestinal solubility. Equilibrium solubility plotted as a 3D topography using the internal center point solubility result for each drug across the $4 \mathrm{CMD}$ matrix. The color shades attached to individual figures for the vertical $z$-axis represent the solubility contour concentration $(\mathrm{mM})$ values for each drug; note, that the solubility ranges vary between figures. (a) Indomethacin, (b) carvedilol, and (c) fenofibrate.

concentration decreases. On the basis of a dose of $200 \mathrm{mg}$, fenofibrate never achieves a BCS high solubility categorization. Also of note for all drugs, is that the center point solubility value is not an average of the low and high solubility window, 
a
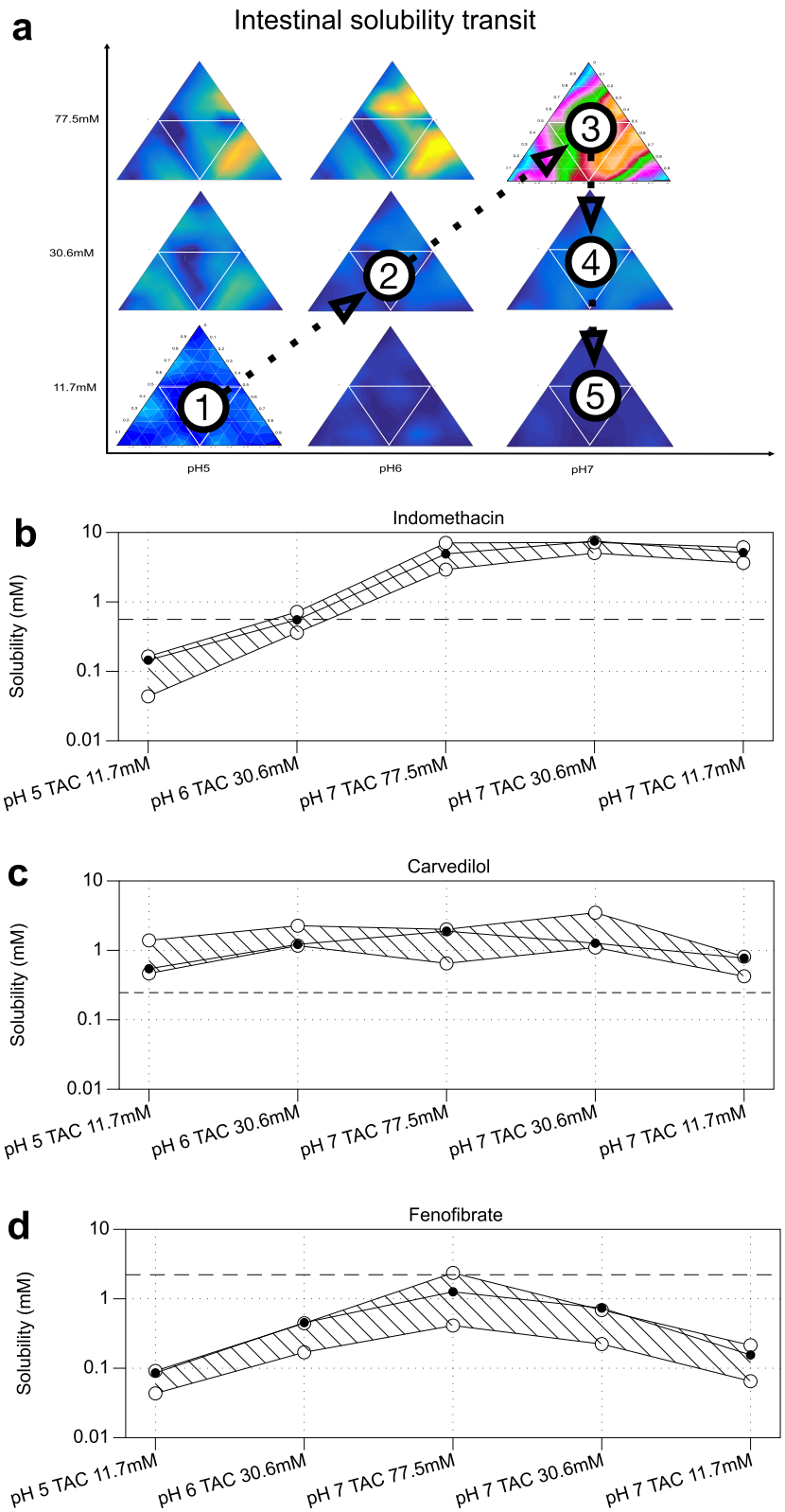

Figure 8. Intestinal equilibrium solubility window using the maximum, minimum, and center internal point solubility results from selected 4CMD experiments (see (a) and Figure 5). TAC, total amphiphile concentration. The dashed line indicates the required solubility for the stated dose dissolved in $250 \mathrm{~mL}$ to be classed as the high solubility with the BCS. (b) Indomethacin, dose = $50 \mathrm{mg}$; (c) carvedilol, dose $=25 \mathrm{mg}$; and (d) fenofibrate, dose $=200 \mathrm{mg}$.

its relative position moves through the various experiments, and each drug displays a different behavior.

\section{DISCUSSION}

General. Each matrix contains 351 solubility measurements, and this is the first comprehensive structured examination of the equilibrium solubility of poorly soluble orally administered drugs covering $\mathrm{pH}$, four amphiphiles, total amphiphile concentration, and amphiphile ratio. The use of a 4CMD matrix is novel and restricts comparison with available literature, where analysis of only single variables in the presence of multiple constant parameters or nonstatistically guided approaches or simply different measurement protocols have been applied. This approach allows for analysis of solubility topography and statistical significance of individual amphiphiles and amphiphile interactions within each 4CMD experiment but with a fixed $\mathrm{pH}$ and total amphiphile concentration. These latter two variables are known to be critical parameters influencing solubility, ${ }^{12,24-26}$ and in order to examine their impact, comparison across the matrix is required which limits statistical analysis since only three data points are available. In addition, the $4 \mathrm{CMD}$ protocol requires analysis of media that are not biorelevant, for example, $100 \mathrm{~mol}$ $\%$ of each amphiphile and amphiphile combinations with extreme and therefore unlikely biological ratios. This will produce solubility measurements that are likely to be unusual, see the Impact of Amphiphile Number on Measured Equilibrium Solubility section and Figure 4. The discussion will therefore only examine gross trends within the presented data and attempt to relate those to prior literature involving the drugs under study or systems where similar variations of media components have been examined. In addition, the parameter values chosen for this study ${ }^{15,31}$ attempt to cover both the fasted $^{13,24}$ and fed ${ }^{12,25}$ states, and therefore, comparison requires to examine solubility data obtained under both conditions.

Overall Solubility Analysis. For all drugs, the published individual solubility measurements in SIFS or HIFs lie within the range created by the lowest (25th percentile) and highest (75th percentile) solubility box line of any of the nine individual $4 \mathrm{CMD}$ experiments. The literature values have been determined using a range of experimental protocols and media compositions, and even with this limitation, the comparability between solubility values demonstrates that the $4 \mathrm{CMD}$ values are in an appropriate range. In addition, the majority of the 4CMD box ranges lie within the lowest and highest solubility whiskers from the fasted and fed $\mathrm{DoE}$ results. There is one exception, the single measurement of carvedilol solubility in fasted HIF, which is lower than the 4CMD lowest box solubility and also lower than the whisker for the fasted DoE solubility range. Also, for carvedilol at the highest total amphiphile concentration, the highest 4CMD box solubility values exceed the highest solubility whiskers from the fasted and fed DoE results. Finally, for all drugs, some of the low solubility 4CMD whiskers extend below the lowest solubility whisker from the fasted DoE result. Overall these results indicate that the $4 \mathrm{CMD}$ matrix is exploring a relevant solubility space encompassing and comparable to published data, with indomethacin and fenofibrate exhibiting excellent compliance, but carvedilol displaying compliance coupled to aberrant behavior at the edges of the investigational space. This is possibly related to the features discussed above (the General section) and the measurement within the matrix of the extreme systems (the Calculated Surface Equilibrium Solubility Contour Plots section) that might be considered to have limited or no biorelevance.

In general, there is an increase in solubility from the lower left corner of the matrix $(\mathrm{pH} 5$ and total amphiphile concentration of $11.7 \mathrm{mM})$ to the upper right $(\mathrm{pH} 7$ and total amphiphile concentration of $77.5 \mathrm{mM}$ ) (Figures 2, 3, and 4). This can be compared to the solubility increase that occurs on shifting from fasted to fed in either sampled or simulated media ${ }^{11,17,31,32}$ and agrees with the published DoE results, which indicate that increased $\mathrm{pH}$ and amphiphile concentration drive solubility. ${ }^{24,26,31}$ In addition, a study using the same four amphiphiles with estradiol indicated that solubility 
was increased as total amphiphile concentration increased, although this only examined three systems at a constant ratio and $\mathrm{pH}^{33}$ Other studies which examined mixed systems ${ }^{22,34}$ have reported the same relationship between drug solubility and total amphiphile concentration, with one reporting ${ }^{22}$ interesting variations between drugs. This variation in solubility behavior related to total amphiphile concentration is also evident in these results; for example, some carvedilol systems in Figure 5b exhibit a maxima at a total amphiphile concentration of $30.6 \mathrm{mM}$. These comparisons indicate that the $4 \mathrm{CMD}$ matrix is examining a relevant solubility space, and that the systems macroscopic solubility behavior is comparable to literature studies.

The measurement points contain a range of amphiphile ratios and numbers (Figure 1 and Table 1), which allows for a solubility comparison between systems with the same $\mathrm{pH}$ and total amphiphile concentration but varying amphiphile numbers and ratios (Figure 4). There are no comparable literature studies with this type and arrangement of data. The striking general result of this analysis across all three drugs is that systems with one or two amphiphiles exhibit a large degree of solubility variability, which is lower in systems with three amphiphiles and minimum in the systems containing all four amphiphiles. In the majority of the $4 \mathrm{CMD}$ experiments, the center point, which contains all four amphiphiles in an equal ratio, is very close to the mean for the four amphiphile points. In addition, Figure 3 indicates that the four amphiphile points are generally within the distribution box plots. There are exceptions for three of the points (out of a possible 108), with two for fenofibrate at $\mathrm{pH} 5$ and a total amphiphile concentration of $30.6 \mathrm{mM}$ and indomethacin at $\mathrm{pH} 5$ and a total amphiphile concentration of $11.7 \mathrm{mM}$. The behavior of indomethacin is different, with a lower solubility variability at higher $\mathrm{pH}$ values, which can be explained by the ionization of the drug dominating solubility behavior. The data indicate that equilibrium solubility at a constant $\mathrm{pH}$ and total amphiphile concentration is heavily influenced by the number of amphiphiles present and the ratio of the amphiphiles, and reducing the spread of ratios and increasing the total number of amphiphiles reduces the solubility variability. Further research in systems structured to examine this effect will be required to fully determine the impact of this finding.

For each $4 \mathrm{CMD}$ experiment, the data were analyzed for a normal distribution pattern. For indomethacin and carvedilol, two or five, respectively, of the nine 4CMDs do not exhibit normality; whereas, for fenofibrate, all the systems are normally distributed. Deviations from normality have been reported in DoE solubility studies, ${ }^{31,35}$ where it may arise either through the sample pattern induced by the DoE structure and/or the fact that drug solubility is not normally distributed in the sample space. The 4CMD protocol samples uniformly across the space within a tetrahedron (Figure 1) and therefore should report a normal solubility distribution, if present, unlike the DoE, which statistically links low and high values of variables ( $\mathrm{pH}$ and individual amphiphile concentrations). The rationale that "drug solubility is not normally distributed" is supported by HIF characterization studies, which indicate that bile salt and lecithin in the fasted state have skewed concentration distributions, ${ }^{13}$ and HIF solubility studies measuring differences between mean and median solubility values ${ }^{17,36}$ indicate a non-normal solubility distribution. The results of this study, when coupled with DoE results, indicate that both reasons are in operation; in DoE, the majority of distributions are non- normal, ${ }^{31,35}$ while in this case, deviations are limited to carvedilol and two for indomethacin. The DoE studies may therefore overestimate deviations from normality due to the statistical sampling structure, but these deviations are inherently present in some systems evidenced by the behavior of carvedilol in the current $4 \mathrm{CMD}$, the shape of the profiles in Figure 3, and the result in Figure 4, indicating that studies with two amphiphiles and variable ratios will induce solubility variability. Absolute resolution of this issue would require the conduct of studies that randomly sample a particular media space.

Indomethacin. Indomethacin is a weak acid with a $\mathrm{p} K_{\mathrm{a}}$ of 4.5, which means that at the lowest $\mathrm{pH}$ (5), it will be approximately $30 \%$ nonionized and almost fully ionized at the highest $\mathrm{pH}$ (7); solubility is therefore expected to increase due to drug ionization. With increasing $\mathrm{pH}$, increasing solubility is evident along with the impact of the amphiphiles, where solubility variability decreases with increasing $\mathrm{pH}$ at a constant total amphiphile concentration but increases with increasing total amphiphile concentration at the same $\mathrm{pH}$. The solubility of the 4CMD systems at $\mathrm{pH} 7$ as well as $\mathrm{pH} 6$ at $77.5 \mathrm{mM}$ are statistically equivalent, indicating that $\mathrm{pH}$ is the major solubility driver but also that amphiphiles influence solubility. This pattern is consistent with indomethacin DoE results, where, in the fasted system, ${ }^{24}$ the ratio of the oleate standardized effect value to $\mathrm{pH}$ was 9 , but in the fed, ${ }^{25}$ it was 4 , indicating the increased influence of amphiphiles with respect to $\mathrm{pH}$ at higher amphiphile concentrations. New in this data is that increasing the total amphiphile concentration at any $\mathrm{pH}$ increases the solubility variability by lowering the low solubility whisker, indicating that particular amphiphiles, ratios, and or combinations reduce indomethacin solubility, for example, the high oleate ratio internal point in Figure 5 at pH 5.

In Figure 3a, the calculated solubility topographies indicate that if the $\mathrm{pH}$ and amphiphile ratio remains constant, but the total amphiphile concentration is increased, then overall solubility will increase, and the solubility contour profile will not change dramatically. This property is already evident in the literature in several systems that have related solubility to total amphiphile concentration ${ }^{33,34}$ or concentrations of individual amphiphiles ${ }^{8,17}$ and to studies using combinations of synthetic surfactants and simulated media. ${ }^{37}$

If the total amphiphile concentration is constant and the $\mathrm{pH}$ changes, an interesting pattern emerges. As the $\mathrm{pH}$ increases, indomethacin ionization will increase but so too will the ionization of the amphiphile oleic acid, which has a $\mathrm{p} K_{\mathrm{a}}$ value around 5 , while the ionization of bile salt with a $\mathrm{p} K_{\mathrm{a}}$ of 2 will not change substantially. ${ }^{38}$ The impact of ionization is evident in Figure 3a, since the vertices, which represent $100 \mathrm{~mol} \%$ bile salt, are high solubility zones at $\mathrm{pH} 7$ but not at $\mathrm{pH}$ 5, indicating that as indomethacin ionization increases, the interaction with bile salt increases (see also Figure 6a). This interaction may be electrostatic in nature, and it has been reported, that indomethacin permeation in vitro is reduced by bile salts, ${ }^{39}$ providing further evidence for this interaction. In Figure $5 \mathrm{a}$, the increased solubilization by ionization of oleate is evident in the high oleate point at total amphiphile concentrations of 11.7 and $77.5 \mathrm{mM}$. Both amphiphiles have positive standardized effect values as single agents (Figure 6a), a result that was also present in the fasted ${ }^{24}$ and $\mathrm{fed}^{25} \mathrm{DoE}$, where, in addition, a significant positive interaction was reported between the amphiphiles and $\mathrm{pH}$ except for bile 
salt and $\mathrm{pH}$ in the fed system. In the present study, both amphiphiles do exhibit significant positive and one negative interactions (Figure 6d), but in the fasted or fed DoE, no significant interactions between these amphiphiles were determined. The results in Figure $6 \mathrm{~d}$ also indicate that there is a significant positive interaction between phospholipid and bile salt or SO, but in the fasted or fed DoE, no significant interactions between these amphiphiles were determined, although a multiple linear regression on indomethacin in intestinal fluids linked bile salt and the phospholipid. ${ }^{12}$ This analysis indicates that if $\mathrm{pH}$ changes, solubility will increase, but the topography will also change due to changes in amphiphile ionization. In addition, it indicates that the DoE approach may miss subtle interactions between components if swamped by a dominant factor, for example, $\mathrm{pH}$, which in this study is a constant within each 4CMD experiment. Overall the behavior agrees with a previous study on the solubility of indomethacin in HIF that attributed $90 \%$ of the solubility to $\mathrm{pH}$ and $10 \%$ to bile salt and phospholipid. ${ }^{12}$

This results in a solubility topography with the major slope rising from low to high $\mathrm{pH}$, with a lower gradient slope based on total amphiphile concentration (Figure 7a). There are two interesting gross features in the topography, a maximum solubility ridge, that runs between $\mathrm{pH} 7$ and a total amphiphile concentration of $30.6 \mathrm{mM}$ and $\mathrm{pH} 677.5 \mathrm{mM}$, and a solubility valley at $\mathrm{pH} 6$ and $30.6 \mathrm{mM}$. The maximum solubility ridge forms due to the lower solubility at $\mathrm{pH} 7$ and total amphiphile concentration of $77.5 \mathrm{mM}$, indicating that high amphiphile concentrations reduce indomethacin solubility, even although it is fully ionized. On the basis of the results in Figure $8 \mathrm{~b}$, this is not likely to impact indomethacin absorption, but this result is not present in the literature, and if this is a general property of acidic drugs, it would be important. The low solubility trough at $\mathrm{pH} 6$ and $30.6 \mathrm{mM}$ total amphiphile concentration is interesting and evident in the results presented in Figures 3a, $5 \mathrm{a}$, and $7 \mathrm{a}$, where this system behaves in an inconsistent manner when compared to the other total amphiphile concentrations at this $\mathrm{pH}$. Figure 5a indicates that this is a systematic effect since all four amphiphile ratios exhibit the response, and this type of behavior (a solubility minima) related to the amphiphile concentration has been reported previously in the literature with cholesterol containing media and griseofulvin. ${ }^{28}$

Carvedilol. Carvedilol is a weak base with a reported $\mathrm{p} K_{\mathrm{a}}$ between 7.8 and $8.25 ;^{40}$ therefore, throughout the 4CMD pH range, it will be ionized, with a minimum percentage ionized of $90 \%$ at $\mathrm{pH} 7$, if the $\mathrm{pK}_{\mathrm{a}}$ is taken to be 8 . It is also reported to have a low and variable solubility in a range of buffers ${ }^{41}$ and intestinal media systems. ${ }^{17}$ In this study, it displays a different profile to indomethacin (Figure $3 \mathrm{~b}$ ), where neither $\mathrm{pH}$ or total amphiphile concentration markedly influence overall solubility. There is evidence of a $\mathrm{pH}$ effect at the low total amphiphile concentration, where solubility decreases as $\mathrm{pH}$ increases, but this is only statistically significant between $\mathrm{pH} 5$ and 6. Eight of the data sets are statistically equivalent, which covers comparisons within and between 4CMD experiments as grouped by total amphiphile concentration (Figure $2 \mathrm{~b}$ ). In addition, five of the nine 4CMD experiments provide data sets that are not normally distributed, compared to only two for indomethacin and zero for fenofibrate. The solubility range of each 4CMD is also variable, and no pattern is evident, except maybe that $\mathrm{pH} 7$ has a reduced variability at total amphiphile concentrations of 30.6 and $77.5 \mathrm{mM}$, and that the lowest total amphiphile concentration has the lowest overall variability irrespective of $\mathrm{pH}$. The calculated solubility peaks and troughs (Figure 3) shift as both $\mathrm{pH}$ and total amphiphile concentration change, indicating a complex solubility behavior influenced by $\mathrm{pH}$, total amphiphile concentration, and ionization of the amphiphiles.

The statistical significance of the individual amphiphiles (see the Supporting Information) indicates that they all exert a roughly equivalent positive solubility impact, with phospholipid possibly being the most significant. In the published fasted DoE, oleate and lecithin provided a small but statistically significant positive solubility influence, while bile salt had a small but significant negative effect. ${ }^{24}$ In the fed DoE, oleate and bile salt had a significant positive solubility influence, while phospholipid was only just significant. ${ }^{25}$ An alternative DoE study indicated that bile salt had a negative impact on solubility, phospholipid was not significant, and oleate was not studied. $^{26}$ These studies together indicate that, for carvedilol, the amphiphiles influence solubility, but that no single amphiphile predominates over the whole 4CMD matrix. There are only 12 significant amphiphile combinations interactions out of a possible 54 but with an indication of a $\mathrm{pH}$ dependency at the highest total amphiphile concentration. The statistical significance matches the solubility behavior, especially at the total amphiphile concentration of $77.5 \mathrm{mM}$, where, at $\mathrm{pH} 7, \mathrm{SPC}$ and $\mathrm{SO}$ are significant, while at lower $\mathrm{pH}$ values, NATC with GMO or SPC is significant. In the fasted state $\mathrm{DoE}$, the combination of oleate and bile salt had a significant negative solubility influence, while in the fed state, the combination of phospholipid and either oleate or monoglyceride had a significant positive impact. Another study indicated the $\mathrm{pH}$ and bile salt had a positive influence on solubility but did not determine any interaction between the amphiphiles. ${ }^{26}$ This is evident where the significance of the interactions is higher at the highest total amphiphile concentration, and in addition, the influence of $\mathrm{pH}$ on these interactions can be seen. There is literature evidence that the solubility of carvedilol in mixed simulated media and surfactant systems is dependent upon the surfactant, ${ }^{37}$ and in one study of surfactants, NATC ${ }^{42}$ displayed a poorer solubilization than cationic or nonionic surfactants. This behavior is evident in the 4CMD carvedilol results in Figure $3 \mathrm{~b}$ at $\mathrm{pH} 7$ and total amphiphile concentration of $11.7 \mathrm{mM}$, where the low solubility zone is at the three vertices of the tetrahedron, which is 100 mol \% bile salt, but this is reversed at $\mathrm{pH} 5$ and total amphiphile concentration of $77.5 \mathrm{mM}$, where the high solubility zone is now in these vertices.

The two factor interactions between NATC and either GMO or SPC at a total amphiphile concentration of $77.5 \mathrm{mM}$ have the greatest magnitude at $\mathrm{pH} 5$, a lower magnitude at $\mathrm{pH}$ 6 , and are not significant at $\mathrm{pH} 7$. This indicates identification of two, three-way parameter interactions, NATC with $\mathrm{pH}$ and either GMO or SPC; the fact that this is only visible at 77.5 $\mathrm{mM}$ might also indicate that total amphiphile concentration is a fourth factor in this interaction. There is weaker evidence for a similar behavior of SPC with SO when combined with $\mathrm{pH}$ and total amphiphile concentration. The previous DoE studies were not powered to detect three- or four-way parameter interactions, and this is, we believe, the first report of a threeor possibly four-way solubilization interaction in SIFs.

For carvedilol at a constant $\mathrm{pH}$, increasing the total amphiphile concentration induces changes in solubility topography, and if total amphiphile concentration is constant and 
$\mathrm{pH}$ is changed, this induces different changes in the solubility topography. Overall, this indicates that, for carvedilol at either a constant $\mathrm{pH}$ or total amphiphile concentration, the solubility topography changes as other variables change and results in a complex pattern that is difficult to interpret but $\mathrm{pH}$, total amphiphile concentration, and amphiphile ratio dependent. The solubility changes are within a relatively small range, with only a 25 -fold variation based on the lowest 25 th percentile (approximately $0.2 \mathrm{mM}$ ) to the highest 75 th percentile (approximately $5 \mathrm{mM}$ ) (Figure $3 \mathrm{~b}$ ), compared to 100 for fenofibrate (Figure 3c). This results in a solubility topography (Figure $7 \mathrm{~b}$ ) that is very flat at all $\mathrm{pH}$ values and low total amphiphile concentrations but then increases markedly at the highest total amphiphile concentration, with a maximum solubility at $\mathrm{pH} 6$ and $30.6 \mathrm{mM}$ total amphiphile concentration.

Overall, the results indicate that, in this system, carvedilol is displaying a complex solubility behavior (five out of the nine 4CMD experiments produce non-normal solubility distributions) that is influenced by multiple interdependent factors, such as $\mathrm{pH}$, amphiphile ratio, ionization, and total amphiphile concentration. The identification of three- and possibly fourway parameter interactions within the matrix highlights the sensitivity of the analysis and also the complexity of carvedilol's behavior. This is replicating the variable solubility behavior of carvedilol reported in the literature, ${ }^{17,41}$ with no single factor across the 4CMD matrix dominating an outcome that is similar to the fasted ${ }^{24}$ and $\mathrm{fed}^{25} \mathrm{DoE}$ results. Carvedilol is a challenging molecule to examine in these systems!

Fenofibrate. Fenofibrate is a neutral drug and will not undergo changes in ionization. It displays a different profile to both indomethacin and carvedilol, with solubility increasing as total amphiphile concentration increases, with minimal changes due to $\mathrm{pH}$, except at the lowest total amphiphile concentration. All of the 4CMD experiments produce a normal distribution but display very low solubility whiskers, and at the highest total amphiphile concentration, the 75th percentile solubilities are higher than the same value for the fed DoE. The low whiskers are probably, as stated previously, a reflection of the 4CMD sampling profile, while the higher values reflect the high total amphiphile concentration employed, when compared to previous literature ${ }^{43}$ and standard fed-simulated media recipes. Overall, this indicates that fenofibrate is behaving in the 4CMD matrix in a manner that is consistent with published results.

At a constant $\mathrm{pH}$, as the total amphiphile concentration increases, the solubility zones remain in the same position (Figure 3c), although a degree of variation is induced as the solubility increases. This is also evident (see Figure S2b) where, at constant $\mathrm{pH}$, the internal points remain in the same general solubility ranking as total amphiphile concentration increases. A aimilar behavior is evident at $\mathrm{pH} 6$ and 7 as the total amphiphile concentration is increased, although there are changes in the starting locations of the high and low solubility zones. Therefore, for fenofibrate, in a similar manner to indomethacin, the solubility will increase as the total amphiphile concentration increases with minimal changes to topography; the latter feature, as stated above, is already evident in the literature for similar systems $s^{7,17,33,34}$ and studies using combinations of synthetic surfactants and simulated media. ${ }^{37}$

If $\mathrm{pH}$ is changed at a constant total amphiphile concentration, the results are different with some interesting variations to the solubility zones. This change can also be visualized in the internal points (see Figure $S 2 b$ ), where the high SO point has the lowest solubility at $\mathrm{pH} 5$ but is the highest at $\mathrm{pH}$ 7. These results indicate that $\mathrm{pH}$ does not have a major influence on solubility but does impart topographical changes as the amphiphile ionization changes, and the magnitude of this effect is greater at the lower total amphiphile concentrations. The published fed DoE studies ${ }^{25}$ and a full range $\mathrm{DoE}$ study ${ }^{31}$ reported that $\mathrm{pH}$ was not a statistically significant parameter for solubilization but was in the fasted study, ${ }^{24}$ where it had a negative impact. Indicating that the 4CMD matrix is replicating behavior that is present in the published literature.

The statistical significance of the individual amphiphiles (see Figure S3c,d), indicates that NATC consistently exerts a negative effect on solubility, a feature that can be seen in all the 4CMD experiments, where the vertices of the tetrahedron, which is $100 \mathrm{~mol} \% \mathrm{BS}$, are low solubility zones. The remaining amphiphiles generally have a positive solubility impact, with oleate exhibiting the strongest activity with interesting $\mathrm{pH}$ and total amphiphile concentration variations. This concurs with the published fasted, ${ }^{24} \mathrm{fed}^{25}$ and full range ${ }^{31}$ DoE results, where oleate was the amphiphile with the highest standardized effect value on fenofibrate solubility, and bile salt had a negative impact in the fed study but positive in the fasted and full range. However, an alternative DoE containing only bile salt and phospholipid indicated that both had a significant positive effect on solubility. ${ }^{26}$ For the factor interactions, 23 out of 54 are significant, with some interesting changes as total amphiphile concentration and $\mathrm{pH}$ change. The most consistent significant positive interaction is between bile salt and phospholipid, which was also the most significant factor interaction reported in the fasted and full range studies but not significant in the fed study. In the current study, this interaction also exhibits a $\mathrm{pH}$ effect at the highest total amphiphile concentration $(77.5 \mathrm{mM})$ as the magnitude of the value decreases as $\mathrm{pH}$ increases, a result that could be applied to all the interactions since only one remains significant at $\mathrm{pH}$ 7. This is evidence of a three-way interaction ( $\mathrm{pH}$ with bile salt with phospholipid), and possibly even a total amphiphile concentration influence which would provide a four-way interaction. However, the DoE studies report that the interaction of bile salt with $\mathrm{pH}$ and bile salt with lecithin are statistically significant but not lecithin with $\mathrm{pH}$ (except in ref 26), indicating that the level of detail available from any study is linked to the experimental design. One study ${ }^{12}$ has performed a multiple linear regression to determine the factors which influence the solubility of neutral compounds (danazol and nifedipine) in postprandial fluids, indicating that approximately $70 \%$ of the solubility could be ascribed to the factors studied with a combination of bile salt and phospholipid, contributing, for both drugs, approximately $50 \%$ and for nifedipine fatty acids and monoglycerides between 25 and $30 \%$ and $\mathrm{pH}$ approximately $15 \%$. The behavior of fenofibrate in the current 4CMD system is therefore consistent with the published data and reveals further subtle details on the relationship between solubility and media factors.

This is reflected in the overall solubility topography presented in Figure 7c, where the greatest slope arises due to increasing total amphiphile concentration and displays two features that are similar to the behavior of carvedilol and indomethacin. The maximum solubility is at $\mathrm{pH} 6$ and a total amphiphile concentration of $30.6 \mathrm{mM}$, similar to carvedilol, 
indicating that $\mathrm{pH} 7$ and a total amphiphile concentration of $77.5 \mathrm{mM}$ suppress fenofibrate solubility. There is also a solubility minima at $\mathrm{pH} 6$ and a total amphiphile concentration of $30.6 \mathrm{mM}$, which is similar to indomethacin.

Impact of Media Composition. The experimental protocol utilizes four amphiphiles, which are known to influence the equilibrium solubility of poorly soluble drugs. ${ }^{24,25}$ The critical micelle concentration of the amphiphiles (NATC, 8-12 $\mathrm{mM}^{44} \mathrm{SO}, 2.2-4 \mathrm{mM}^{45} \mathrm{GMO}, 0.004$ $\mathrm{mM}^{46}$ and SPC, $\left.0.4 \mathrm{mM}^{47}\right)$ is below the lowest total amphiphile concentration $(11.7 \mathrm{mM})$; therefore, the media within the matrix will be a colloidal system that will consist of a range of structures (micelles, mixed micelles, and vesicles), ${ }^{33,48-50}$ which will change as molar ratio (within a $4 \mathrm{CMD}$ changes), concentration, and $\mathrm{pH}$ (across the matrix) vary. This study did not examine colloidal structures, but the results indicate that changes in these structures are influencing solubility (Figure 3). Other research groups have presented solubility inflections related to amphiphile concentration ${ }^{43}$ or to the presence of solubility maxima and minima as the ratio of components changes ${ }^{28,43}$ behavior that is also evident in this study.

One interesting feature of the results, visible in Figure 7, is that the $\mathrm{pH} 6$ and total amphiphile concentration of $30.6 \mathrm{mM}$ system produces an unusual solubility result for indomethacin and carvedilol that is lower than the other two $\mathrm{pH} 6$ systems and results in an negative inflection of the lines in Figure 5. There is not a large solubility variation in this system (Figure 4 ), implying that this is an inherent property of this set of conditions. With only three $\mathrm{pH}$ values and total amphiphile concentrations, it is not possible to fully examine the interesting behavior of the $30.6 \mathrm{mM}$ system. This is worthy of further study since it may represent a set of conditions with unusual colloidal properties or where the colloidal structures inhibit solubility.

Intestinal Equilibrium Solubility Window. The current study was designed to provide a matrix that would reveal the solubility behavior of poorly soluble drugs across a range of simulated media compositions and to investigate the impact of media components. However, it is also known, that the composition of intestinal fluids will change as a result of prandial status, anatomical position within the small intestine, ${ }^{12,14-16}$ and in relation to inter- and intra-individual variability. ${ }^{13}$ In order to apply this information, compartmental model systems of the gastrointestinal tract have been proposed, ${ }^{51,52}$ with some models proposing up to seven different compartments for the small intestine. ${ }^{53}$ In this study, each $4 \mathrm{CMD}$ experiment represents a set of conditions that could be related to intestinal compartments or fluid compositions, as a material was exposed to different prandial states and anatomical locations. A possible scenario (Figure 8a) utilizes five of the nine experiments, the minimum and maximum solubility values of the internal points have been employed since these are more realistic solubility determinations. However, this probably underestimates solubility variability based on the 25 th and 75 th percentile range in Figure 3. This is similar to the use of early, middle, and late simulated fasted media systems to represent different phases. $^{12,20}$

The analysis (Figure 8) indicates that the drugs exhibit three different profiles. For indomethacin, the impact of $\mathrm{pH}$ is obvious, and once the system is above $\mathrm{pH} \mathrm{6}$, solubility is sufficient to be classed as high within the BCS system. While for carvedilol, a constant solubility window is evident that is not influenced by $\mathrm{pH}$ or total amphiphile concentration, and which is sufficient to class the drug as high solubility. For fenofibrate, a further profile type is evident, with solubility reaching a maxima linked to the total amphiphile concentration and, even at the maxima, not attaining sufficient solubility to be classed as high. This type of analysis could be useful for pharmacokinetic predictions and formulation development, but it must, at this stage, be applied cautiously, since it does not allow for dynamic changes in composition ${ }^{12,14}$ or rate of movement down the intestinal tract. ${ }^{54}$ Of interest is that the position and profile of the center point relative to the maximum and minimum internal point values are also different for the three drugs and do not lie directly in the middle of the solubility window. Indicating that a single point equilibrium solubility measurement will not allow for possible variability or indicate its position with respect to the overall solubility range.

\section{CONCLUSIONS}

This is the first 4CMD study of equilibrium solubility using biorelevant amphiphiles (NATC, SPC, SO, and GMO) measured within a matrix of three $\mathrm{pH}$ values $(5,6$, and 7$)$ and three total amphiphile concentrations $(11.7,30.6$, and 77.5 $\mathrm{mM}$ ) providing a solubility matrix covering the fasted and fed intestinal media states with visualization of the solubility topography. The macroscopic solubility behavior within the matrix is consistent with the published literature in either the fasted or fed intestinal fluid, ${ }^{17}$ simulated fasted or fed intestinal fluid, ${ }^{30}$ and with statistically based equilibrium solubility determinations $^{24-26,31}$ or multiple linear regression. ${ }^{12}$ The 4CMD matrix is therefore examining a relevant equilibrium solubility space, although this may not be reflected in other physicochemical properties, such as dissolution.

Overall solubility increases from low $\mathrm{pH}$ and total amphiphile concentration to high $\mathrm{pH}$ and total amphiphile concentrations in a similar manner to switching from fasted to fed media. ${ }^{35}$ The behavior of the exemplar acidic (indomethacin), basic (carvedilol), and neutral (fenofibrate) drugs is in agreement with the published $\mathrm{DoE}^{24-26}$ and other statistical studies. ${ }^{12}$ For acidic compounds, $\mathrm{pH}$ is the major solubility driver, while for basic and neutral drugs, a combination of $\mathrm{pH}$ and amphiphiles contributes to the solubility. This approach therefore provides an orthogonal verification of the published literature findings. In addition, this study identifies, for the first time, three and four factor interactions for two of the drugs involving $\mathrm{pH}, \mathrm{NATC}$, and SPC with total amphiphile concentration as a fourth factor. Determination of this degree of interaction has not been previously possible. The results indicate that all three drugs behave in a different fashion at any point within the matrix and reveal that the complex solubility topography previously reported using a single 4CMD condition $^{29}$ extends throughout intestinal media conditions irrespective of drug, $\mathrm{pH}$, or amphiphile concentration. The study conclusively demonstrates that the topography arises due to the combination of drug, $\mathrm{pH}$, and amphiphile in terms of number present, total concentration, ratio, and when linked to $\mathrm{pH}$ ionization; all of which will be applicable in both simulated and sampled intestinal fluids.

The 4CMD statistical design provides a unique view on the behavior of simulated media systems, indicating that the greater the number of amphiphiles included within the system, the lower the measured solubility variability. In addition, the analysis of solubility distributions for normality (Figure 2) 
indicates that the 4CMD's uniform sampling structure determines a lower number of non-normal distributions than DoE studies. ${ }^{31}$ These finding have profound implications for the design of simulated media systems in terms of the number of amphiphiles included, the ratios employed, and the arrangement of sampling points. It appears, therefore, that further statistical refinement is required to find a model suited to the particular issues and intricacies of intestinal fluid.

The results demonstrate that solubility variability is an inherent property of these systems, and this reinforces our previous discussion that "a single point solubility measurement in a fixed composition media will provide a value that might be situated in a valley, plateau, slope, or peak". ${ }^{29}$ Therefore, in either simulated media or sampled intestinal fluids, the utility of a single solubility measurement is limited without a knowledge of the surrounding solubility topography. In the research literature, the issue of solubility variability is an ever present problem that seems to have no resolution, and in this study, the three drugs investigated produce a different solubility topography implying that every drug will be unique. However, the "scaleable" topography behavior of indomethacin and fenofibrate in certain situations indicates that structured behavior is present, and that guidelines, if not eventually rules, are possible if a sufficient knowledge of the systems can be obtained. This will not be simple, in this study, carvedilol displays a behavior that is not easily interpreted, other than too many factors are influencing solubility, which swamps the applied statistical methodology.

In a previous publication, ${ }^{29}$ it was suggested that the discrete nature of each individual 4CMD experiment could be tailored to provide an equilibrium solubility window that could be applied to PBPK compartmental models of the gastrointestinal tract. The matrix in the current study was designed to cover intestinal conditions; however, by judicious choice of 4CMD experiments from within the matrix, it does permit a possible visualization of equilibrium solubility in multiple discrete compartments and indicates the feasibility of this approach. The result that the three drugs have three different profiles also highlights the issues associated with a single point solubility determination. Future, 4CMD matrices could be specifically designed to mimic and study possible gastrointestinal compartments for PBPK modeling.

The 4CMD matrix approach is resource intensive and not easily applied during drug development or even typical research studies, unless high throughput systems can be developed. The current design can only handle four components, and it could be argued, that other amphiphiles should be present, for example, cholesterol ${ }^{13,28}$ and/or lysolecithin, ${ }^{23}$ which would increase the number of measurements required. The results in Figure 4 indicate that the impact on equilibrium solubility of additional components as "single" factors in a one or two factor media system would be high; whereas, in a three or four factor media system, this would not (unless drug specific effects are present) be pronounced. Judicious application of this finding would reduce the number of solubility measurements required. Further work will be required to refine the approach so that the number of samples can be reduced with the removal of $100 \mathrm{~mol} \%$ points, two or three amphiphile mixtures, for example, while a relevant range of conditions $(\mathrm{pH}$, number of amphiphiles, ratios, and concentrations) are investigated. However, this is likely to run into the simple statistical limitation that decreasing the sample number limits the experiment's statistical certainty and resolution. $^{31}$

\section{ASSOCIATED CONTENT}

\section{S Supporting Information}

The Supporting Information is available free of charge on the ACS Publications website at DOI: 10.1021/acs.molpharmaceut.8b01238.

Basic physicochemical information on the three drug compounds employed within the four component mixture design, information on the three HPLC methods employed to analyze the equilibrium solubility of the drugs in the media after incubation, influence of amphiphile number on measured equilibrium solubility results for carvedilol and fenofibrate, internal point results for carvedilol and fenofibrate, and standardized effect values for individual amphiphiles for carvedilol and fenofibrate and two amphiphile interactions for carvedilol and fenofibrate (PDF)

\section{AUTHOR INFORMATION}

\section{Corresponding Author}

*Telephone: +44(0) 141548 2454; Fax: +44(0) 141548 4903; E-mail: g.w.halbert@strath.ac.uk.

ORCID $\odot$

Gavin W. Halbert: 0000-0001-8553-3647

Notes

The authors declare no competing financial interest.

\section{ACKNOWLEDGMENTS}

The authors gratefully acknowledge the financial support of the Oral Biopharmaceutical Tools (OrBiTo) (115369), European Union Innovative Medicines Initiative Program and the assistance and input of the multiple colleagues associated with this project. GWH and JP are funded by Cancer Research UK (C149/A20496 and C149/A20740 respectively).

\section{ABBREVIATIONS:}

BCS, Biopharmaceutics Classification System; DoE, design of experiment; FaSSIF, fasted simulated intestinal fluid; FeSSIF, fed simulated intestinal fluid; IVIVC, in vitro in vivo correlation; 4CMD, 4-component mixture design; NATC, sodium taurocholate; SPC, soya phosphatidyl choline; GMO, glyceryl mono-oleate; SO, sodium oleate

\section{REFERENCES}

(1) Koziolek, M.; Grimm, M.; Schneider, F.; Jedamzik, P.; Sager, M.; Kuhn, J. P.; Siegmund, W.; Weitschies, W. Navigating the human gastrointestinal tract for oral drug delivery: Uncharted waters and new frontiers. Adv. Drug Delivery Rev. 2016, 101, 75-88.

(2) Sugano, K.; Okazaki, A.; Sugimoto, S.; Tavornvipas, S.; Omura, A.; Mano, T. Solubility and dissolution profile assessment in drug discovery. Drug Metab. Pharmacokinet. 2007, 22 (4), 225-254.

(3) Amidon, G. L.; Lennernas, H.; Shah, V. P.; Crison, J. R. A Theoretical Basis For a Biopharmaceutic Drug Classification - the Correlation Of In-Vitro Drug Product Dissolution and In-Vivo Bioavailability. Pharm. Res. 1995, 12 (3), 413-420.

(4) Lipinski, C. A. Drug-like properties and the causes of poor solubility and poor permeability. J. Pharmacol. Toxicol. Methods 2000, 44 (1), 235-249.

(5) Dressman, J. B.; Vertzoni, M.; Goumas, K.; Reppas, C. Estimating drug solubility in the gastrointestinal tract. Adv. Drug Delivery Rev. 2007, 59 (7), 591-602. 
(6) Lindahl, A.; Ungell, A. L.; Knutson, L.; Lennernas, H. Characterization of fluids from the stomach and proximal jejunum in men and women. Pharm. Res. 1997, 14 (4), 497-502.

(7) Pedersen, B. L.; Brondsted, H.; Lennernas, H.; Christensen, F. N.; Mullertz, A.; Kristensen, H. G. Dissolution of hydrocortisone in human and simulated intestinal fluids. Pharm. Res. 2000, 17 (2), 183189.

(8) Pedersen, B. L.; Mullertz, A.; Brondsted, H.; Kristensen, H. G. A comparison of the solubility of danazol in human and simulated gastrointestinal fluids. Pharm. Res. 2000, 17 (7), 891-894.

(9) Vertzoni, M.; Pastelli, E.; Psachoulias, D.; Kalantzi, L.; Reppas, C. Estimation of intragastric solubility of drugs: in what medium? Pharm. Res. 2007, 24 (5), 909-17.

(10) Kalantzi, L.; Goumas, K.; Kalioras, V.; Abrahamsson, B.; Dressman, J. B.; Reppas, C. Characterization of the human upper gastrointestinal contents under conditions simulating bioavailability/ bioequivalence studies. Pharm. Res. 2006, 23 (1), 165-76.

(11) Clarysse, S.; Brouwers, J.; Tack, J.; Annaert, P.; Augustijns, P. Intestinal drug solubility estimation based on simulated intestinal fluids: comparison with solubility in human intestinal fluids. Eur. J. Pharm. Sci. 2011, 43 (4), 260-9.

(12) Clarysse, S.; Psachoulias, D.; Brouwers, J.; Tack, J.; Annaert, P.; Duchateau, G.; Reppas, C.; Augustijns, P. Postprandial changes in solubilizing capacity of human intestinal fluids for BCS class II drugs. Pharm. Res. 2009, 26 (6), 1456-66.

(13) Riethorst, D.; Mols, R.; Duchateau, G.; Tack, J.; Brouwers, J.; Augustijns, P. Characterization of Human Duodenal Fluids in Fasted and Fed State Conditions. J. Pharm. Sci. 2016, 105, 673-681.

(14) de la Cruz-Moreno, M. P.; Montejo, C.; Aguilar-Ros, A.; Dewe, W.; Beck, B.; Stappaerts, J.; Tack, J.; Augustijns, P. Exploring drug solubility in fasted human intestinal fluid aspirates: Impact of interindividual variability, sampling site and dilution. Int. J. Pharm. 2017, $528(1-2), 471-484$.

(15) Bergstrom, C. A.; Holm, R.; Jorgensen, S. A.; Andersson, S. B.; Artursson, P.; Beato, S.; Borde, A.; Box, K.; Brewster, M.; Dressman, J.; Feng, K. I.; Halbert, G.; Kostewicz, E.; McAllister, M.; Muenster, U.; Thinnes, J.; Taylor, R.; Mullertz, A. Early pharmaceutical profiling to predict oral drug absorption: current status and unmet needs. Eur. J. Pharm. Sci. 2014, 57, 173-99.

(16) Fuchs, A.; Dressman, J. B. Composition and Physicochemical Properties of Fasted-State Human Duodenal and Jejunal Fluid: A Critical Evaluation of the Available Data. J. Pharm. Sci. 2014, 103 (11), 3398-3411.

(17) Augustijns, P.; Wuyts, B.; Hens, B.; Annaert, P.; Butler, J.; Brouwers, J. A review of drug solubility in human intestinal fluids: Implications for the prediction of oral absorption. Eur. J. Pharm. Sci. 2014, 57C, 322-332.

(18) Galia, E.; Nicolaides, E.; Horter, D.; Lobenberg, R.; Reppas, C.; Dressman, J. B. Evaluation of various dissolution media for predicting in vivo performance of class I and II drugs. Pharm. Res. 1998, 15 (5), 698-705.

(19) Dressman, J. B.; Amidon, G. L.; Reppas, C.; Shah, V. P. Dissolution testing as a prognostic tool for oral drug absorption: Immediate release dosage forms. Pharm. Res. 1998, 15 (1), 11-22.

(20) Bou-Chacra, N.; Melo, K. J. C.; Morales, I. A. C.; Stippler, E. S.; Kesisoglou, F.; Yazdanian, M.; Lobenberg, R. Evolution of Choice of Solubility and Dissolution Media After Two Decades of Biopharmaceutical Classification System. AAPS J. 2017, 19 (4), 989-1001.

(21) Markopoulos, C.; Andreas, C. J.; Vertzoni, M.; Dressman, J.; Reppas, C. In-vitro simulation of luminal conditions for evaluation of performance of oral drug products: Choosing the appropriate test media. Eur. J. Pharm. Biopharm. 2015, 93, 173-82.

(22) Kleberg, K.; Jacobsen, F.; Fatouros, D. G.; Mullertz, A. Biorelevant media simulating fed state intestinal fluids: colloid phase characterization and impact on solubilization capacity. J. Pharm. Sci. 2010, 99 (8), 3522-32.

(23) Fuchs, A.; Leigh, M.; Kloefer, B.; Dressman, J. B. Advances in the design of fasted state simulating intestinal fluids: FaSSIF-V3. Eur. J. Pharm. Biopharm. 2015, 94, 229-40.
(24) Khadra, I.; Zhou, Z.; Dunn, C.; Wilson, C. G.; Halbert, G. Statistical investigation of simulated intestinal fluid composition on the equilibrium solubility of biopharmaceutics classification system class II drugs. Eur. J. Pharm. Sci. 2015, 67, 65-75.

(25) Zhou, Z.; Dunn, C.; Khadra, I.; Wilson, C. G.; Halbert, G. W. Statistical investigation of simulated fed intestinal media composition on the equilibrium solubility of oral drugs. Eur. J. Pharm. Sci. 2017, 99, 95-104.

(26) Madsen, C. M.; Feng, K. I.; Leithead, A.; Canfield, N.; Jorgensen, S. A.; Mullertz, A.; Rades, T. Effect of composition of simulated intestinal media on the solubility of poorly soluble compounds investigated by design of experiments. Eur. J. Pharm. Sci. 2018, 111, 311.

(27) Bevernage, J.; Brouwers, J.; Clarysse, S.; Vertzoni, M.; Tack, J.; Annaert, P.; Augustijns, P. Drug supersaturation in simulated and human intestinal fluids representing different nutritional states. $J$. Pharm. Sci. 2010, 99 (11), 4525-34.

(28) Khoshakhlagh, P.; Johnson, R.; Langguth, P.; Nawroth, T.; Schmueser, L.; Hellmann, N.; Decker, H.; Szekely, N. K. Fasted-State Simulated Intestinal Fluid "FaSSIF-C", a Cholesterol Containing Intestinal Model Medium for In Vitro Drug Delivery Development. J. Pharm. Sci. 2015, 104, 2213.

(29) Zhou, Z.; Dunn, C.; Khadra, I.; Wilson, C. G.; Halbert, G. W. Influence of Physiological Gastrointestinal Surfactant Ratio on the Equilibrium Solubility of BCS Class II Drugs Investigated Using a Four Component Mixture Design. Mol. Pharmaceutics 2017, 14 (12), $4132-4144$.

(30) Soderlind, E.; Karlsson, E.; Carlsson, A.; Kong, R.; Lenz, A.; Lindborg, S.; Sheng, J. J. Simulating Fasted Human Intestinal Fluids: Understanding the Roles of Lecithin and Bile Acids. Mol. Pharmaceutics 2010, 7 (5), 1498-1507.

(31) Perrier, J.; Zhou, Z.; Dunn, C.; Khadra, I.; Wilson, C. G.; Halbert, G. Statistical investigation of the full concentration range of fasted and fed simulated intestinal fluid on the equilibrium solubility of oral drugs. Eur. J. Pharm. Sci. 2018, 111, 247-256.

(32) Fagerberg, J. H.; Tsinman, O.; Sun, N.; Tsinman, K.; Avdeef, A.; Bergstrom, C. A. S. Dissolution Rate and Apparent Solubility of Poorly Soluble Drugs in Biorelevant Dissolution Media. Mol. Pharmaceutics 2010, 7 (5), 1419-1430.

(33) Ilardia-Arana, D.; Kristensen, H. G.; Mullertz, A. Biorelevant dissolution media: aggregation of amphiphiles and solubility of estradiol. J. Pharm. Sci. 2006, 95 (2), 248-55.

(34) Magee, G. A.; French, J.; Gibbon, B.; Luscombe, C. Bile salt/ lecithin mixed micelles optimized for the solubilization of a poorly soluble steroid molecule using statistical experimental design. Drug Dev. Ind. Pharm. 2003, 29 (4), 441-50.

(35) Ainousah, B. E.; Perrier, J.; Dunn, C.; Khadra, I.; Wilson, C. G.; Halbert, G. Dual Level Statistical Investigation of Equilibrium Solubility in Simulated Fasted and Fed Intestinal Fluid. Mol. Pharmaceutics 2017, 14 (12), 4170-4180.

(36) Psachoulias, D.; Vertzoni, M.; Goumas, K.; Kalioras, V.; Beato, S.; Butler, J.; Reppas, C. Precipitation in and supersaturation of contents of the upper small intestine after administration of two weak bases to fasted adults. Pharm. Res. 2011, 28 (12), 3145-58.

(37) Yang, B.; Wu, C.; Ji, B.; Ai, X.; Kuang, X.; Wu, M.; Sun, M.; Luo, C.; He, Z.; Sun, J. The biorelevant concentration of Tween 80 solution is a simple alternative medium to simulated fasted state intestinal fluid. RSC Adv. 2015, 5 (127), 104846-104853.

(38) Holm, R.; Mullertz, A.; Mu, H. Bile salts and their importance for drug absorption. Int. J. Pharm. 2013, 453 (1), 44-55.

(39) Riethorst, D.; Brouwers, J.; Motmans, J.; Augustijns, P. Human intestinal fluid factors affecting intestinal drug permeation in vitro. Eur. J. Pharm. Sci. 2018, 121, 338-346.

(40) Alves, J. M. V.; Prado, L. D.; Rocha, H. V. A. Evaluation and correlation of the physicochemical properties of carvedilol. Pharm. Dev. Technol. 2016, 21 (7), 856-866.

(41) Hamed, R.; Awadallah, A.; Sunoqrot, S.; Tarawneh, O.; Nazzal, S.; AlBaraghthi, T.; Al Sayyad, J.; Abbas, A. pH-Dependent Solubility 
and Dissolution Behavior of Carvedilol-Case Example of a Weakly Basic BCS Class II Drug. AAPS PharmSciTech 2016, 17 (2), 418-26.

(42) Chakraborty, S.; Shukla, D.; Jain, A.; Mishra, B.; Singh, S. Assessment of solubilization characteristics of different surfactants for carvedilol phosphate as a function of pH. J. Colloid Interface Sci. 2009, 335 (2), 242-9.

(43) Zangenberg, N. H.; Mullertz, A.; Kristensen, H. G.; Hovgaard, L. A dynamic in vitro lipolysis model II: Evaluation of the model. Eur. J. Pharm. Sci. 2001, 14 (3), 237-244.

(44) Meyerhoffer, S. M.; McGown, L. B. Critical micelle concentration behavior of sodium taurocholate in water. Langmuir 1990, 6 (1), 187-191.

(45) Joshi, H. D.; Desai, C. M. SPECTROPHOTOMETRIC DETERMINATION OF CRITICAL MICELLE CONCENTRATION OF SURFACTANTS WITH DYE INDICATORS. Indian J. Chem. 1994, 33 (10), 965-966.

(46) Milak, S.; Zimmer, A. Glycerol monooleate liquid crystalline phases used in drug delivery systems. Int. J. Pharm. 2015, 478 (2), 569-87.

(47) A.P.L., Inc. https://avantilipids.com/tech-support/physicalproperties/cmcs (accessed Nov 23, 2018).

(48) Konyakhina, T. M.; Wu, J.; Mastroianni, J. D.; Heberle, F. A.; Feigenson, G. W. Phase diagram of a 4-component lipid mixture: DSPC/DOPC/POPC/chol. Biochim. Biophys. Acta, Biomembr. 2013, 1828 (9), 2204-14.

(49) Elvang, P. A.; Hinna, A. H.; Brouwers, J.; Hens, B.; Augustijns, P.; Brandl, M. Bile Salt Micelles and Phospholipid Vesicles Present in Simulated and Human Intestinal Fluids: Structural Analysis by Flow Field-Flow Fractionation/Multiangle Laser Light Scattering. J. Pharm. Sci. 2016, 105 (9), 2832-9.

(50) Elvang, P. A.; Jacobsen, A. C.; Bauer-Brandl, A.; Stein, P. C.; Brandl, M. Co-existing colloidal phases in artificial intestinal fluids assessed by AF4/MALLS and DLS: A systematic study into cholate \& (lyso-) phospholipid blends, incorporating celecoxib as a model drug. Eur. J. Pharm. Sci. 2018, 120, 61-72.

(51) Yu, L. X.; Amidon, G. L. A compartmental absorption and transit model for estimating oral drug absorption. Int. J. Pharm. 1999, 186 (2), 119-125.

(52) Gobeau, N.; Stringer, R.; De Buck, S.; Tuntland, T.; Faller, B. Evaluation of the GastroPlus Advanced Compartmental and Transit (ACAT) Model in Early Discovery. Pharm. Res. 2016, 33 (9), 212639.

(53) Rowland, M.; Peck, C.; Tucker, G. Physiologically-based pharmacokinetics in drug development and regulatory science. Annu. Rev. Pharmacol. Toxicol. 2011, 51, 45-73.

(54) Hens, B.; Corsetti, M.; Spiller, R.; Marciani, L.; Vanuytsel, T.; Tack, J.; Talattof, A.; Amidon, G. L.; Koziolek, M.; Weitschies, W.; Wilson, C. G.; Bennink, R. J.; Brouwers, J.; Augustijns, P. Exploring gastrointestinal variables affecting drug and formulation behavior: Methodologies, challenges and opportunities. Int. J. Pharm. 2017, 519 (1-2), 79-97. 